\title{
Deep inelastic scattering cross sections from the gauge/string duality
}

\author{
Ezequiel Koile, Nicolas Kovensky and Martin Schvellinger \\ IFLP-CCT-La Plata, CONICET and Departamento de Física, \\ Universidad Nacional de La Plata, \\ Calle 49 y 115, C.C. 67, (1900) La Plata, Buenos Aires, Argentina. \\ E-mail: koile@fisica.unlp.edu.ar, nico.koven@fisica.unlp.edu.ar, \\ martin@fisica.unlp.edu.ar
}

AbSTRACT: Differential cross sections of deep inelastic scattering of charged leptons from hadrons are investigated by using the gauge/string duality. We consider vector mesons derived from different holographic dual models obtaining a general expression. We focus on the strongly coupled regime of dual gauge theories for different values of the Bjorken parameter. We find new predictions which are particularly interesting for differential scattering cross sections of polarized leptons scattered off polarized vector mesons. We also carry out a detailed comparison of the moments of the structure functions with lattice QCD results.

KEYWords: Gauge-gravity correspondence, D-branes, AdS-CFT Correspondence

ARXIV EPRINT: 1507.07942 


\section{Contents}

1 Introduction 1

2 Deep inelastic scattering cross section 4

$\begin{array}{lll}3 & \text { DIS differential cross sections and structure functions } & 6\end{array}$

$\begin{array}{ll}3.1 \text { The general case } & 6\end{array}$

3.2 Hadron polarizations $\quad 9$

$\begin{array}{lll}3.3 & \text { Helicity amplitudes } & 12\end{array}$

4 DIS differential cross sections from the gauge/string duality $\quad 14$

4.1 Range A: $1 / \sqrt{\lambda} \ll x<1 \quad 16$

4.2 Range B: $e^{-\sqrt{\lambda}} \ll x \ll 1 / \sqrt{\lambda} \quad 19$

4.3 Range C: $x \sim e^{-\sqrt{\lambda}} \quad 21$

5 Results and discussion $\quad 22$

5.1 Structure functions and differential cross sections 22

$\begin{array}{lll}5.2 & \text { Comparison with lattice QCD } & 28\end{array}$

\section{Introduction}

In a recent series of papers [1-3] we have studied deep inelastic scattering (DIS) of charged leptons from scalar mesons and from polarized vector mesons at strong coupling by considering different holographic dual models based on flavor Dp-branes in the probe approximation. In those papers we have studied the planar limit of dual gauge theories corresponding to D3D7-, D4D6 $\overline{\mathrm{D} 6}$ - and D4D8 $\overline{\mathrm{D} 8}$-brane models $\left([4,5]\right.$ and $[6]$, respectively). ${ }^{1}$ In $[1-3]$ we have investigated the hadronic tensor and, for polarized vector mesons, we have obtained the corresponding eight structure functions. We have derived general relations, i.e. modelindependent relations, of the Callan-Gross type between different structure functions. This is very interesting because from that one can infer a sort of universal behavior which would also be expected for QCD in the planar limit. This universal property holds because, for all the probe flavor Dp-brane models we have considered, mesons are described as fluctuations of flavor Dp-branes in terms of the corresponding Dirac-Born-Infeld action. In particular, in [1] we have studied DIS from dynamical holographic scalar and polarized vector mesons with one flavor, by considering the flavored Dp-brane models of references [4-6]. Moreover, in [2] we have carried out a non-trivial extension of these results for multi-flavored mesons in the planar limit and with the condition $N_{f} \ll N$, where $N$ denotes the number of colors and $N_{f}$ the number of flavors. In that paper we also have worked out the corresponding next-to-leading order Lagrangians in the $1 / N$ and $N_{f} / N$ expansions. In [1,2] we have

\footnotetext{
${ }^{1}$ The calculations carried out in [1-3] are inspired in the methods developed in reference [7], where DIS of charged leptons from glueballs in the planar limit of $N=1^{*}$ SYM theory has been considered [8].
} 
focused on the Bjorken parameter regime where $1 / \sqrt{\lambda} \ll x<1$, and therefore the calculations have been done by using the gauge/string duality in terms of the type IIA and type IIB supergravity dual descriptions of the gauge theories in the large $N$ limit and at strong 't Hooft coupling $\lambda$.

In a more recent work [3] we have studied DIS of charged leptons from hadrons in the $e^{-\sqrt{\lambda}} \ll x \ll 1 / \sqrt{\lambda}$ and $x \sim e^{-\sqrt{\lambda}}$ regimes. In [3] we have focused on single-flavored scalar and vector mesons in the planar limit. This has been investigated in terms of different holographic dual models with flavor Dp-branes in type IIA and type IIB superstring theories [4-6]. We have calculated the hadronic tensor and the structure functions for scalar and polarized vector mesons. In particular, for polarized vector mesons we have obtained the eight structure functions at small values of the Bjorken parameter.

In the present work we further extend our previous results by studying DIS cross sections of charged leptons from polarized vector mesons and carrying out a qualitative comparison between moments of structure functions calculated by using gauge/string duality methods and available lattice QCD data. We emphasize that the character of the comparison which we perform is qualitative since these structure functions have been obtained in the context of the large- $N$ and large- $\lambda$ limits of confining gauge theories derived from their string theory dual models, which in fact do not lead to real QCD. At this point we can briefly describe some important differences between QCD and the holographic dual models we consider. We can start from the D3D7-brane model, which is the holographic dual description corresponding to the planar limit of the strongly coupled $\mathrm{SU}(N) \mathcal{N}=2$ supersymmetric Yang-Mills theory with fundamental quarks [4]. Obviously, this is not the large $N$ limit of QCD but it is related to it. On the other hand, we study the $\mathrm{D} 4 \mathrm{D} 8 \overline{\mathrm{D}} 8$-brane model developed in [6] which at low energy is expected to be within the same universality class as the planar limit of QCD. At high energy this model behaves in a different way compared with QCD, since in that regime the Kaluza-Klein modes generated by the compatification of the $x^{4}$-direction of the $N$ D4-branes along the $S^{1}$ become relevant. Therefore these additional degrees of freedom, which are obviously absent in QCD, play a role in the dynamics of this dual gauge theory at high energy. In addition, this model [6] enjoys an $\mathrm{SO}(5)$ global symmetry, which is not present in QCD. Thus, one can say that these Dp-brane models do not lead to holographic dual gauge theories with exactly the same properties as QCD in the planar limit. However, these holographic dual models represent infrared confining gauge theories and share dynamical properties with QCD at low energy. For instance, the Sakai-Sugimoto model realizes spontaneous chiral symmetry breaking. Also, the chiral Lagrangian has been derived from this model [6].

We also ought to mention that the comparison we carry out between our results and phenomenology unfortunately has the disadvantage that our calculations and the existing phenomenology and lattice QCD calculations belong to different parametric regimes. On the one hand, in our previous papers [1-3] we have done calculations within the Regge region. The asymptotic Regge region is relevant since it dominates total cross sections and differential cross sections when considering small angle. However, in the asymptotic Regge regime it is not possible to perform quantum field theory (QFT) calculations, neither one can use lattice QCD methods in order to obtain QCD amplitudes [9]. From this discussion 
one infers that it is difficult to make any quantitative comparison of these models and $\mathrm{SU}(3)$ QCD data. In this way our aim is to compare qualitatively certain aspects of the phenomenology derived from these dual models with QCD. At the end, by taking into account all these caveats, we would like to see how our results compare with lattice QCD data. Indeed, this kind of comparison has proved to be qualitatively (and even in certain cases quantitatively) interesting in several situations as we shall explain in the last section of this paper. In fact, from a theoretical perspective it has given some important insights about the behavior of gauge theories in general, in the strong coupling regime where perturbative QFT methods cannot be used. This assertion is indeed very important because given a certain solution of supergravity and/or superstring theory, in principle, the gauge/string duality can be used to define a strongly coupled holographic dual $\mathrm{SU}(N)$ gauge theory in the large $N$ limit. Suppose in addition that such a QFT has composite states. A natural way to study these states is in terms of the DIS calculations as described in $[1-3,7,9]$. Therefore, the interest of these methods goes beyond the large $N$ limit of QCD itself, i.e. it is useful for any other confining gauge theory with a string theory holographic dual model.

This paper is organized as follows. In section 2 we introduce and derive a number of relevant expressions necessary to construct the DIS cross sections of charged leptons from hadrons of spin $s=0, \frac{1}{2}$ and 1 in a generic form. This is a fully general expression for the DIS differential cross section which holds for any infrared confining gauge theory at any value of the rank of the gauge group $N$ (i.e. beyond the planar limit), and at any value of the coupling. In section 3 we focus on the case of polarized vector mesons and we present these derivations in detail. Thus, in this section we derive the DIS cross section from leptons off vector mesons which are unpolarized, longitudinally polarized, transversally polarized and with partial polarization. We also study the corresponding helicity amplitudes of the forward Compton scattering directly related to the DIS process and discuss on physical implications.

In section 4 we derive general (i.e. model independent) expressions for the DIS cross sections at large 't Hooft coupling, in the three mentioned regimes of the Bjorken parameter. In general terms the hadronic tensor can be derived from the operator product expansion (OPE) of two electromagnetic currents inside the hadron. At weak 't Hooft coupling the OPE is dominated by approximate twist-two operators, and it corresponds to the scattering of a lepton from a weakly interacting parton. However, in this section we consider the strong coupling limit. In this case the OPE which leads to the hadronic tensor is dominated by double-trace operators, and it corresponds to the scattering of a lepton from the entire hadron. Within this strongly coupled regime we consider the parametric range $1 / \sqrt{\lambda} \ll x<1$ where supergravity describes DIS. Furthermore, we consider the case where $e^{-\sqrt{\lambda}} \ll x \ll 1 / \sqrt{\lambda}$, in which excited strings are produced. Also, we study the case for $x \sim e^{-\sqrt{\lambda}}$, where the produced excited strings have a size comparable to the scale of the AdS space.

In section 5 we discuss our results derived in the previous sections such as differential cross sections in terms of the Bjorken parameter and the fractional energy loss of the lepton, as well as the structure functions and their moments, and carry out a comparison 


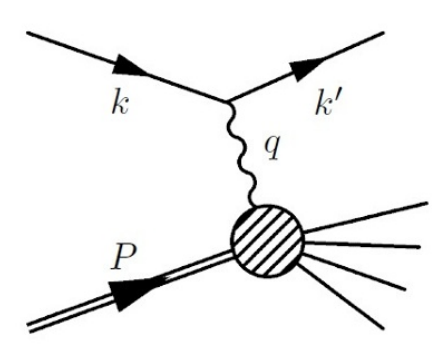

Figure 1. Schematic representation of DIS. A lepton with momentum $k$ interacts with a hadron with momentum $P$ through the exchange of a virtual photon with momentum $q$.

with moments of structure functions for the pion and the $\rho$ meson obtained from lattice QCD. It turns out that our results of the first three moments of the $F_{2}$-structure function of the pion and the $F_{1}$-structure function of the $\rho$ meson are in very good quantitative agreement with the corresponding ones from lattice QCD.

\section{Deep inelastic scattering cross section}

In this section we first introduce some definitions of kinematical variables which will be useful for the rest of the work. Then, we introduce the leptonic and hadronic tensors and give an expression for the DIS differential cross section which holds for any value of the coupling.

The DIS process is schematically represented in figure 1.

We consider the following definitions which are the same as in reference $[10]^{2}$

- $M$ is the mass of the hadron, being the on-shell condition $M^{2}=-P^{2}$.

- $E$ is the energy of the incident lepton.

- $k$ is the momentum of the incident lepton $k^{\mu}=(E, 0,0, E)$.

- $E^{\prime}$ is the energy of the scattered lepton.

- $k^{\prime}$ is the momentum of the scattered lepton $k^{\prime \mu}=E^{\prime}(1, \sin \theta \cos \phi, \sin \theta \sin \phi, \cos \theta)$.

- $P$ is the momentum of the hadron $P^{\mu}=(M, 0,0,0)$ in the rest frame. ${ }^{3}$

- $q$ is the momentum transfer or momentum of the virtual photon $q=k-k^{\prime}$.

- $\nu$ is the energy loss of the lepton $\nu=E-E^{\prime}=-\frac{P \cdot q}{M}$.

- $y$ is the fractional energy loss of the lepton $y=\frac{\nu}{E}=\frac{P \cdot q}{P \cdot k}$.

- $x$ is Bjorken parameter $x=-\frac{q^{2}}{2 P \cdot q}$.

- $t$ is a parameter defined as $t=\frac{P^{2}}{q^{2}}$.

In what follows we have assumed that the leptons are almost massless.

\footnotetext{
${ }^{2}$ Notice however that we use the mostly-plus metric $\eta^{\mu \nu}=\operatorname{diag}(-,+,+,+)$.

${ }^{3}$ Fixed-target experiment.
} 
The DIS differential cross section can be written in terms of the so-called leptonic and hadronic tensors $l^{\mu \nu}$ and $W^{\mu \nu}$, respectively. Thus, we have

$$
\frac{d^{2} \sigma}{d x d y d \phi}=\frac{e^{4}}{16 \pi^{2} q^{4}} y l^{\mu \nu} W_{\mu \nu}
$$

where $e$ denotes the electron charge. The leptonic tensor depends on the incident lepton beam and it can be easily derived from perturbative QED, leading to

$$
\begin{aligned}
l_{\frac{1}{2}}^{\mu \nu} & =\left[2\left(k^{\mu} k^{\prime \nu}+k^{\nu} k^{\prime \mu}\right)-2 \eta^{\mu \nu} k \cdot k^{\prime}\right]+\left[-2 i \epsilon^{\mu \nu \alpha \beta} q_{\alpha} s_{l \beta}\right], \quad m_{l}^{2} \sim 0 \\
& =l_{\mathrm{sym}}^{\mu \nu}+l_{\mathrm{ant}}^{\mu \nu},
\end{aligned}
$$

where $l_{\mathrm{sym}}^{\mu \nu}$ and $l_{\text {ant }}^{\mu \nu}$ denote the symmetric and antisymmetric parts, respectively. $s_{l}^{\mu}$ is the lepton spin four-vector defined by boosting $\left(0, \vec{s}_{l}\right)$ from the rest frame, or equivalently by using the spinor states $u$ and $\bar{u}$ which leads to

$$
s_{l}^{\mu}=\bar{u}\left(k, s_{l}\right) \gamma^{\mu} \gamma_{5} u\left(k, s_{l}\right) .
$$

On the other hand, the hadronic tensor describes the internal structure of the hadron. It cannot be calculated by using perturbative QCD because there are soft processes involved. In this paper we focus on spin-one hadrons since they have a very rich structure given in terms of eight structure functions which follow from the most general Lorentztensor decomposition of the hadronic tensor. We also write the results of the structure functions for the case of scalar mesons since we shall discuss them in section 5 .

In general terms, the hadronic tensors for a spin $s=1, \frac{1}{2}$ and 0 can be written as follows

$$
\begin{aligned}
& W_{\mu \nu}^{1}=W_{\mu \nu}^{\mathrm{SF}}+W_{\mu \nu}^{\mathrm{Sb}}+W_{\mu \nu}^{\mathrm{Ag}}, \\
& W_{\mu \nu}^{\frac{1}{2}}=W_{\mu \nu}^{\mathrm{SF}}+W_{\mu \nu}^{\mathrm{Ag}} \\
& W_{\mu \nu}^{0}=W_{\mu \nu}^{\mathrm{SF}}
\end{aligned}
$$

where

$$
\begin{aligned}
W_{\mu \nu}^{\mathrm{SF}} & =F_{1} \eta_{\mu \nu}-\frac{F_{2}}{P \cdot q} P_{\mu} P_{\nu}, \\
W_{\mu \nu}^{\mathrm{Sb}} & =b_{1} r_{\mu \nu}-\frac{b_{2}}{6}\left(s_{\mu \nu}+t_{\mu \nu}+u_{\mu \nu}\right)-\frac{b_{3}}{2}\left(s_{\mu \nu}-u_{\mu \nu}\right)-\frac{b_{4}}{2}\left(s_{\mu \nu}-t_{\mu \nu}\right), \\
W_{\mu \nu}^{\mathrm{Ag}} & =-\frac{i g_{1}}{P \cdot q} \epsilon_{\mu \nu \lambda \sigma} q^{\lambda} s^{\sigma}-\frac{i g_{2}}{(P \cdot q)^{2}} \epsilon_{\mu \nu \lambda \sigma} q^{\lambda}\left(P \cdot q s^{\sigma}-s \cdot q P^{\sigma}\right) .
\end{aligned}
$$

The superscripts refer to the symmetry properties under the exchange of Lorentz indices ( $S$ and $A$ ) and to the usual name given to the structure functions involved in each term $\left(F_{i}\right.$ 's, $b_{i}$ 's and $g_{i}$ 's). In addition, we have used the following definitions [11]

$$
\begin{aligned}
& r_{\mu \nu} \equiv \frac{1}{(P \cdot q)^{2}}\left(q \cdot \zeta^{*} q \cdot \zeta-\frac{1}{3}(P \cdot q)^{2} \kappa\right) \eta_{\mu \nu}, \\
& s_{\mu \nu} \equiv \frac{2}{(P \cdot q)^{3}}\left(q \cdot \zeta^{*} q \cdot \zeta-\frac{1}{3}(P \cdot q)^{2} \kappa\right) P_{\mu} P_{\nu},
\end{aligned}
$$




$$
\begin{aligned}
t_{\mu \nu} & \equiv \frac{1}{2(P \cdot q)^{2}}\left[-\frac{4}{3}(P \cdot q) P_{\mu} P_{\nu}+\left(\left(q \cdot \zeta^{*}\right)\left(P_{\mu} \zeta_{\nu}+P_{\nu} \zeta_{\mu}\right)+c . c .\right)\right], \\
u_{\mu \nu} & \equiv \frac{1}{P \cdot q}\left[\left(\zeta_{\mu}^{*} \zeta_{\nu}+\zeta_{\nu}^{*} \zeta_{\mu}\right)-\frac{2}{3} M^{2} \eta_{\mu \nu}-\frac{2}{3} P_{\mu} P_{\nu}\right], \\
s^{\sigma} & \equiv \frac{-i}{M^{2}} \epsilon^{\sigma \alpha \beta \rho} \zeta_{\alpha}^{*} \zeta_{\beta} P_{\rho}, \\
\kappa & =1-4 x^{2} t
\end{aligned}
$$

where $\zeta^{\mu}$ is the polarization vector of the hadron. Then, one can write a general expression for the differential cross section of the DIS of a charged lepton from a hadron in terms of the symmetric and antisymmetric parts of these tensors

$$
\frac{d^{2} \sigma}{d x d y d \phi}=\frac{e^{4}}{16 \pi^{2} q^{4}} y\left[l_{\mathrm{sym}}^{\mu \nu} W_{\mu \nu}^{\mathrm{SF}}+l_{\mathrm{ant}}^{\mu \nu} W_{\mu \nu}^{\mathrm{Ag}}+l_{\mathrm{sym}}^{\mu \nu} W_{\mu \nu}^{\mathrm{Sb}}\right]
$$

where $\phi$ is the azimuthal angle. The differential cross section depends trivially on $\phi$ but non-trivially on $x$ and $y$, which can be chosen as two independent parameters describing the DIS process, together with the spin polarizations of the lepton and the hadron. It is worth to note that in the case of spin-zero hadrons only the first term in the square bracket of eq. (2.16) contributes, while for the spin- $1 / 2$ case the second term also appears. The last term contributes for spin-one hadrons and contains four additional $b_{i}$ structure functions.

\section{DIS differential cross sections and structure functions}

In this section we derive the DIS differential cross sections of charged leptons scattered from hadrons with spin $s=0, \frac{1}{2}$ and 1 . We consider first the general case, and then we study different polarization states of the hadron beam.

\subsection{The general case}

Let us begin with the first term of eq. (2.16), which is present in hadrons with spin $0, \frac{1}{2}$ and 1. We obtain the following term expressed in terms of the $F_{1}$ and $F_{2}$ structure functions

$$
\begin{aligned}
\frac{e^{4}}{16 \pi^{2} q^{4}} \text { y } l_{\mathrm{sym}}^{\mu \nu} W_{\mu \nu}^{\mathrm{SF}} & =\frac{e^{4} M E}{4 \pi^{2} q^{4}}\left\{x y^{2} F_{1}+\left[1-y+x^{2} y^{2} t\right] F_{2}\right\} \\
& \simeq \frac{e^{4} M E}{4 \pi^{2} q^{4}}\left\{x y^{2} F_{1}+[1-y] F_{2}\right\},
\end{aligned}
$$

where in the last step we have approximated $|t| \simeq 0 .{ }^{4}$ The second term, which appears for both $s=\frac{1}{2}$ and $s=1$ spins is given by

$$
\begin{aligned}
\frac{e^{4}}{16 \pi^{2} q^{4}} y l_{\mathrm{ant}}^{\mu \nu} W_{\mu \nu}^{\mathrm{Ag}}=\frac{e^{4} M E}{4 \pi^{2} q^{4}}\{ & y^{2} g_{1}\left[2 x \frac{\left(s_{h} \cdot s_{l}\right)}{(p \cdot q)}+\frac{\left(q \cdot s_{h}\right)\left(q \cdot s_{l}\right)}{(p \cdot q)^{2}}\right] \\
+ & \left.2 x y^{2} g_{2}\left[\frac{\left(s_{h} \cdot s_{l}\right)}{(p \cdot q)}-\frac{\left(q \cdot s_{h}\right)\left(p \cdot s_{l}\right)}{(p \cdot q)^{2}}\right]\right\} .
\end{aligned}
$$

\footnotetext{
${ }^{4}$ This is the equation (5.2) of reference [10]. An extra factor $2 \pi$ appears in the denominator because we are calculating $\frac{d^{2} \sigma}{d x d y d \phi}$ as in [10]. In reference [11], on the other hand, it has been calculated $\frac{d^{2} \sigma}{d x d y}=2 \pi \frac{d^{2} \sigma}{d x d y d \phi}$.
} 
This term depends on the structure functions $g_{1}$ and $g_{2}$. Notice that in the expression for the first term we contract only symmetric factors, while in the expression for the second one only antisymmetric tensors are contracted. In addition, in the last calculation we have used the identity $\epsilon^{\mu \nu \alpha \beta} \epsilon_{\mu \nu \lambda \sigma}=-2\left(\eta_{\lambda}^{\alpha} \eta_{\sigma}^{\beta}-\eta_{\sigma}^{\alpha} \eta_{\lambda}^{\beta}\right)$.

Let us now calculate the most general form of the third term which only appears for spin-one hadrons, and can be written in terms of the $b_{i}$ structure functions. Firstly, we need to calculate the contractions

$$
l_{\mathrm{sym}}^{\mu \nu} r_{\mu \nu} ; \quad l_{\mathrm{sym}}^{\mu \nu} s_{\mu \nu} ; \quad l_{\mathrm{sym}}^{\mu \nu} t_{\mu \nu} ; \quad l_{\mathrm{sym}}^{\mu \nu} u_{\mu \nu} .
$$

In order to simplify the notation we define a pseudo-scalar product $\langle\cdot \cdot\rangle$ given by

$$
\left\langle k_{1} k_{2}\right\rangle \equiv \frac{3}{2} \frac{\left(k_{1} \cdot \zeta^{*}\right)\left(k_{2} \cdot \zeta\right)+c . c .}{(P \cdot q)^{2}},
$$

where c.c. indicates complex conjugate. This pseudo-scalar product is linear on both sides and it is also commutative. In addition, since we take the $|t| \ll 1$ limit we set $\kappa=1$. Then, we obtain

$$
\begin{aligned}
l_{\mathrm{sym}}^{\mu \nu} r_{\mu \nu}= & {\left[2\left(k^{\mu} k^{\prime \nu}+k^{\nu} k^{\prime \mu}\right)-2 \eta^{\mu \nu} k \cdot k^{\prime}\right] \frac{1}{(P \cdot q)^{2}}\left(q \cdot \zeta^{*} q \cdot \zeta-\frac{1}{3}(P \cdot q)^{2}\right) \eta_{\mu \nu} } \\
= & -\frac{4}{3}\left(k \cdot k^{\prime}\right)[\langle q q\rangle-1] \\
= & \frac{4}{3} M E x y[\langle q q\rangle-1] \\
l_{\mathrm{sym}}^{\mu \nu} s_{\mu \nu}= & {\left[2\left(k^{\mu} k^{\prime \nu}+k^{\nu} k^{\prime \mu}\right)-2 \eta^{\mu \nu} k \cdot k^{\prime}\right] \frac{2}{(P \cdot q)^{3}}\left(q \cdot \zeta^{*} q \cdot \zeta-\frac{1}{3}(P \cdot q)^{2}\right) P_{\mu} P_{\nu} } \\
= & \frac{4}{3}\left[\frac{2(P \cdot k)\left(P \cdot k^{\prime}\right)}{(P \cdot q)}-\frac{P^{2}\left(k \cdot k^{\prime}\right)}{(P \cdot q)}\right][\langle q q\rangle-1] \\
= & -\frac{8}{3} M E[\langle q q\rangle-1]\left[\frac{1}{y}-1+x^{2} y t\right] \\
l_{\mathrm{sym}}^{\mu \nu} t_{\mu \nu}= & {\left[2\left(k^{\mu} k^{\prime \nu}+k^{\nu} k^{\prime \mu}\right)-2 \eta^{\mu \nu} k \cdot k^{\prime}\right] \frac{1}{2(P \cdot q)^{2}} \times } \\
& {\left[-\frac{4}{3}(P \cdot q) P_{\mu} P_{\nu}+\left(\left(q \cdot \zeta^{*}\right)\left(P_{\mu} \zeta_{\nu}+P_{\nu} \zeta_{\mu}\right)+c . c .\right)\right] } \\
= & \frac{-1}{\langle q q\rangle-1} l_{\mathrm{sym}}^{\mu \nu} s_{\mu \nu}+\frac{4}{3}\left\langle q k^{\prime}\right\rangle(k \cdot P)+\frac{4}{3}\langle q k\rangle\left(k^{\prime} \cdot P\right) \\
= & \frac{4}{3} M E\left[2\left(\frac{1}{y}-1+x^{2} y t\right)-\left\langle q k^{\prime}\right\rangle-\langle q k\rangle(1-y)\right]
\end{aligned}
$$

and

$$
\begin{aligned}
l_{\mathrm{sym}}^{\mu \nu} u_{\mu \nu} & =\left[2\left(k^{\mu} k^{\prime \nu}+k^{\nu} k^{\prime \mu}\right)-2 \eta^{\mu \nu} k \cdot k^{\prime}\right] \frac{1}{(P \cdot q)}\left[\left(\zeta_{\mu}^{*} \zeta_{\nu}+\zeta_{\nu}^{*} \zeta_{\mu}\right)-\frac{2}{3} M^{2} \eta_{\mu \nu}-\frac{2}{3} P_{\mu} P_{\nu}\right] \\
& =\left[\frac{4}{3}\left\langle k k^{\prime}\right\rangle(P \cdot q)-2 \frac{M^{2}\left(k \cdot k^{\prime}\right)}{(P \cdot q)}\right]-\frac{2 M^{2}}{(P \cdot q)[\langle q q\rangle-1]} l_{\mathrm{sym}}^{\mu \nu} r_{\mu \nu}-\frac{1}{\langle q q\rangle-1} l_{\mathrm{sym}}^{\mu \nu} s_{\mu \nu} \\
& =\frac{8}{3} M E\left[-y\left\langle k k^{\prime}\right\rangle+\left(\frac{1}{y}-1\right)+2 x^{2} y t\right] .
\end{aligned}
$$


Finally, by writing the cross section contribution from the $b_{i}$ structure functions as $\alpha_{1} b_{1}+$ $\alpha_{2} b_{2}+\alpha_{3} b_{3}+\alpha_{4} b_{4}$ one finds the following expressions for the $\alpha_{i}$ coefficients

$$
\begin{aligned}
\alpha_{1}= & \frac{e^{4}}{16 \pi^{2} q^{2}} y l_{\mathrm{sym}}^{\mu \nu} r_{\mu \nu}=\frac{M E e^{4}}{2^{2} 3 \pi^{2} q^{4}} x y^{2}[\langle q q\rangle-1] \\
\alpha_{2}= & -\frac{e^{4}}{16 \pi^{2} q^{4}} \frac{y}{6} l_{\mathrm{sym}}^{\mu \nu}\left(s_{\mu \nu}+t_{\mu \nu}+u_{\mu \nu}\right) \\
= & \frac{M E e^{4}}{2^{3} 3^{2} \pi^{2} q^{4}} \times \\
& {\left[2(\langle q q\rangle-3)(1-y)+y\left\langle q k^{\prime}\right\rangle+y(1-y)\langle q k\rangle+2 y^{2}\left\langle k k^{\prime}\right\rangle-2(4-\langle q q\rangle) x^{2} y^{2} t\right] } \\
\alpha_{3}= & -\frac{e^{4}}{16 \pi^{2} q^{4}} \frac{y}{2} l_{\mathrm{sym}}^{\mu \nu}\left(s_{\mu \nu}-u_{\mu \nu}\right) \\
= & \frac{M E e^{4}}{2^{2} 3 \pi^{2} q^{4}}\left[(1-y)\langle q q\rangle-y^{2}\left\langle k k^{\prime}\right\rangle+x^{2} y^{2} t(\langle q q\rangle+1)\right] \\
\alpha_{4}= & -\frac{e^{4}}{16 \pi^{2} q^{4}} \frac{y}{2} l_{\mathrm{sym}}^{\mu \nu}\left(s_{\mu \nu}-t_{\mu \nu}\right) \\
= & -\frac{M E e^{4}}{2^{3} 3 \pi^{2} q^{4}}\left[2(1-y)\langle q q\rangle-y\left\langle q k^{\prime}\right\rangle-\langle q k\rangle y(1-y)+2\langle q q\rangle x^{2} y^{2} t\right]
\end{aligned}
$$

By using the linearity of $\langle\cdot \cdot\rangle$ we obtain

$$
\begin{aligned}
& \frac{e^{4}}{16 \pi^{2} q^{4}} \text { y } l_{\mathrm{sym}}^{\mu \nu} W_{\mu \nu}^{\mathrm{Sb}}= \\
& \frac{M E e^{4}}{12 \pi^{2} q^{4}}\left\{b_{1} x y^{2}[\langle q q\rangle-1]+\frac{1}{6}\left[(2-3 y)\langle q q\rangle+y(2-3 y)\langle q k\rangle+2 y^{2}\langle k k\rangle-6(1-y)\right] b_{2}\right. \\
& \left.+\left[(1-y)\langle q q\rangle-y^{2}\langle k k\rangle+y^{2}\langle q k\rangle\right] b_{3}+\frac{1}{2}[(2-y)\langle q q\rangle+y(-2+y)\langle q k\rangle] b_{4}+\mathcal{O}(t)\right\} .
\end{aligned}
$$

Now, in the $\sqrt{-t} \ll 1$ limit we can relate the values of $\langle q q\rangle,\langle q k\rangle$, and $\langle k k\rangle$, since in this limit $\frac{q^{\mu}}{q^{0}}=\frac{k^{\mu}}{E}$, where the Lorentz index $\mu$ runs from 0 to 3 . Then, in the hadron rest frame we obtain the relations

$$
\begin{aligned}
\frac{q^{\mu} q^{\nu}}{M^{2}\left(q^{0}\right)^{2}} & =\frac{\frac{1}{2}\left(q^{\mu} k^{\nu}+k^{\mu} q^{\nu}\right)}{M^{2} q^{0} E}=\frac{k^{\mu} k^{\nu}}{M^{2} E^{2}}, \\
2 \frac{q^{\mu} q^{\nu}}{(P \cdot q)^{2}} \zeta_{\mu} \zeta_{\nu}^{*} & =\frac{\left(q^{\mu} k^{\nu}+k^{\mu} q^{\nu}\right)}{(P \cdot q)(P \cdot k)} \zeta_{\mu} \zeta_{\nu}^{*}=2 \frac{k^{\mu} k^{\nu}}{(P \cdot k)^{2}} \zeta_{\mu} \zeta_{\nu}^{*}, \\
\frac{3}{2} \frac{\left(q \cdot \zeta^{*}\right)(q \cdot \zeta)+c . c .}{(P \cdot q)^{2}} & =\frac{(P \cdot q)}{(P \cdot k)} \frac{3}{2} \frac{\left(q \cdot \zeta^{*}\right)(k \cdot \zeta)+c . c .}{(P \cdot q)^{2}}=\frac{(P \cdot q)^{2}}{(P \cdot k)^{2}} \frac{3}{2} \frac{\left(k \cdot \zeta^{*}\right)(k \cdot \zeta)+c . c .}{(P \cdot q)^{2}},
\end{aligned}
$$

and

$$
\langle q q\rangle=y\langle q k\rangle=y^{2}\langle k k\rangle .
$$

Thus, for $\sqrt{-t} \ll 1$ the part of the cross section associated with the $b_{i}$ structure functions of the hadronic tensor reduces to a simpler form

$$
\frac{e^{4}}{16 \pi^{2} q^{4}} \text { y } l_{\mathrm{sym}}^{\mu \nu} W_{\mu \nu}^{\mathrm{Sb}}=\frac{M E e^{4}}{2^{2} 3 \pi^{2} q^{4}}(\langle q q\rangle-1)\left[b_{1} x y^{2}+b_{2}(1-y)\right]+\mathcal{O}(\sqrt{-t}) .
$$


Notice that in this limit the contributions to the cross section coming from $b_{3}$ and $b_{4}$ are sub-leading in $t$ even if $b_{3}$ and $b_{4}$ are not necessarily sub-leading themselves. Thus, the full DIS differential cross section from spin-one hadrons to this order becomes

$$
\begin{aligned}
\frac{d \sigma_{\text {spin } 1}}{d x d y d \phi}= & \frac{M E e^{4}}{4 \pi^{2} q^{4}}\left\{\left[x y^{2} F_{1}+(1-y) F_{2}\right]+\frac{1}{3}(\langle q q\rangle-1)\left[x y^{2} b_{1}+(1-y) b_{2}\right]\right. \\
& \left.+y^{2} g_{1}\left[2 x \frac{\left(s_{h} \cdot s_{l}\right)}{(p \cdot q)}+\frac{\left(q \cdot s_{h}\right)\left(q \cdot s_{l}\right)}{(p \cdot q)^{2}}\right]+2 x y^{2} g_{2}\left[\frac{\left(s_{h} \cdot s_{l}\right)}{(p \cdot q)}-\frac{\left(q \cdot s_{h}\right)\left(p \cdot s_{l}\right)}{(p \cdot q)^{2}}\right]\right\} \\
= & \frac{M E e^{4}}{4 \pi^{2} q^{4}}\left\{x y^{2}\left[F_{1}+\frac{1}{3}(\langle q q\rangle-1) b_{1}\right]+(1-y)\left[F_{2}+\frac{1}{3}(\langle q q\rangle-1) b_{2}\right]\right. \\
& \left.+y^{2} g_{1}\left[2 x \frac{\left(s_{h} \cdot s_{l}\right)}{(p \cdot q)}+\frac{\left(q \cdot s_{h}\right)\left(q \cdot s_{l}\right)}{(p \cdot q)^{2}}\right]+2 x y^{2} g_{2}\left[\frac{\left(s_{h} \cdot s_{l}\right)}{(p \cdot q)}-\frac{\left(q \cdot s_{h}\right)\left(p \cdot s_{l}\right)}{(p \cdot q)^{2}}\right]\right\} .
\end{aligned}
$$

\subsection{Hadron polarizations}

Now, we calculate the dimensionless factor

$$
\langle q q\rangle=\frac{3}{2} \frac{\left(q \cdot \zeta^{*}\right)(q \cdot \zeta)+\text { c.c. }}{(P \cdot q)^{2}}=3 \frac{q^{\mu} q^{\nu}}{(P \cdot q)^{2}} \zeta_{\mu} \zeta_{\nu}^{*},
$$

for different polarizations of the hadron in order to obtain the DIS differential cross section $\frac{d \sigma}{d x d y d \phi}$ for the particular cases which may be phenomenologically relevant, namely: when the hadron beam is unpolarized, longitudinally polarized, transversally polarized and also we study partial polarizations of the hadron beam. In all cases the longitudinal or $\hat{z}$-axis is defined by the spatial components of $k$, in other words, the direction of the incident lepton beam.

Unpolarized hadron beam. In order to describe unpolarized hadron beams we have to average over polarizations obtaining

$$
\overline{\zeta_{\mu} \zeta_{\nu}^{*}}=\frac{1}{3}\left(\eta_{\mu \nu} M^{2}+P_{\mu} P_{\nu}\right)
$$

Then,

$$
\langle q q\rangle_{\text {unpol }}=\langle\overline{q q}\rangle=\frac{1}{(P \cdot q)^{2}}\left[M^{2} q^{2}+(P \cdot q)^{2}\right]=1-4 x^{2} t=\kappa \simeq 1,
$$

thus, we obtain a null contribution from the $b_{i}$ structure functions as expected for an unpolarized target [11]. In particular, for a longitudinally polarized leptonic beam we have $s_{l}=H_{l} k$ with $H_{l}= \pm 1$ for positive and negative helicity, respectively. Then, we obtain

$$
\frac{d \sigma}{d x d y d \phi}=\frac{M E e^{4}}{4 \pi^{2} q^{4}}\left\{x y^{2} F_{1}+(1-y) F_{2}\right\} .
$$

Longitudinal polarization. Now, we consider the case when the hadron beam is polarized in the direction of the incident beam and call it $\hat{z}$-axis. Thus, we can take the polarization to be

$$
\zeta_{L}=\frac{M}{\sqrt{2}}\left(0,1, i H_{h}, 0\right) \quad \rightarrow \quad s^{\sigma}=H_{h} M \hat{z}
$$


where $H_{h}= \pm 1$ indicates that the hadron polarization is parallel or anti-parallel to the beam direction, respectively. Then, we obtain the following expressions

$$
\begin{aligned}
\left(q \cdot \zeta_{L}\right) & =-\frac{M E^{\prime} \sin \theta}{\sqrt{2}} e^{i H_{h} \phi}, \\
\left(q \cdot \zeta_{L}^{*}\right)\left(q \cdot \zeta_{L}\right) & =\frac{M^{2} E^{\prime 2} \sin ^{2} \theta}{2}=\frac{q^{2} M^{2}}{2}\left[(1-y)+t x^{2} y^{2}\right], \\
\langle q q\rangle_{\mathrm{LP}} & =\frac{3}{2} \frac{1}{(P \cdot q)^{2}} M^{2} E^{\prime 2} \sin ^{2} \theta=-6 x^{2} t\left[(1-y)+t x^{2} y^{2}\right] \simeq 0 .
\end{aligned}
$$

In this case the factor $\langle q q\rangle-1$ only gives a minus sign. In particular, for a longitudinally polarized lepton beam we obtain

$$
\frac{d \sigma}{d x d y d \phi}=\frac{M E e^{4}}{4 \pi^{2} q^{4}}\left\{x y^{2}\left[F_{1}-\frac{1}{3} b_{1}\right]+(1-y)\left[F_{2}-\frac{1}{3} b_{2}\right]-H_{l} H_{h}(2-y) y x g_{1}+\mathcal{O}(t)\right\} .
$$

Transversal polarization. Since the system under consideration has azimuthal symmetry we can choose any polarization for the hadron in the $(\hat{x}, \hat{y})$-plane. For transversal polarization we choose for example the $\hat{x}$-axis and set

$$
\zeta_{T}=\frac{M}{\sqrt{2}}\left(0,0,1, i H_{h}\right) \quad \rightarrow \quad s^{\sigma}=H_{h} M \hat{x} ; \quad H_{h}= \pm 1 .
$$

Then, we obtain

$$
\begin{aligned}
\left(q \cdot \zeta_{T}\right) & =\frac{M^{2}}{\sqrt{2}}\left[\sqrt{\frac{(1-y)}{-t}-x^{2} y^{2}} \sin \phi+i H_{h}\left(-\frac{1}{2 x t}+x y\right)\right] \\
& \simeq \frac{M^{2}}{\sqrt{2}}\left[\sqrt{\frac{(1-y)}{-t}} \sin \phi-\frac{i H_{h}}{2 x t}\right] \\
\left(q \cdot \zeta_{T}^{*}\right)\left(q \cdot \zeta_{T}\right) & =\frac{M^{4}}{2 t^{2}}\left[\frac{1}{4 x^{2}}-t(1-y) \sin ^{2} \phi\right] \\
\langle q q\rangle_{\mathrm{TP}} & =\frac{3}{2} \frac{1}{(P \cdot q)^{2}} \frac{M^{4}}{t^{2}}\left[\frac{1}{4 x^{2}}-t(1-y) \sin ^{2} \phi\right] \\
& =6 x^{2}\left[\frac{1}{4 x^{2}}-t(1-y) \sin ^{2} \phi\right] \simeq \frac{3}{2}
\end{aligned}
$$

and the dimensionless factor becomes $\langle q q\rangle-1 \approx \frac{1}{2}$. In particular, for a longitudinally polarized lepton beam we obtain

$$
\begin{aligned}
\frac{d \sigma}{d x d y d \phi}=\frac{M E e^{4}}{4 \pi^{2} q^{4}}\{ & x y^{2}\left[F_{1}+\frac{1}{6} b_{1}\right]+(1-y)\left[F_{2}+\frac{1}{6} b_{2}\right] \\
& \left.+H_{l} H_{h} 2 x^{2} \sqrt{-t} \sqrt{1-y}\left(y g_{1}+2 g_{2}\right) \cos \phi\right\} .
\end{aligned}
$$


Partial polarizations. The $b_{i}$ structure function terms in eq. (3.13) and in particular the dimensionless factor $\langle q q\rangle-1$ can be written in a more general form

$$
\frac{e^{4}}{16 \pi^{2} q^{4}} y l_{\mathrm{sym}}^{\mu \nu} W_{\mu \nu}^{\mathrm{Sb}}=\frac{M E e^{4}}{2 \sqrt{3} \pi^{2} q^{4}} \operatorname{Tr}\left(\rho \cdot \lambda_{8}\right)\left[b_{1} x y^{2}+b_{2}(1-y)\right]+\mathcal{O}(\sqrt{-t}),
$$

where $\lambda_{8}=\frac{1}{\sqrt{3}} \operatorname{diag}(1,1,-2)$ and $\rho$ is the spin-one density matrix that accounts for the possibility of having a statistical production of the hadron beam. Notice that

$$
\operatorname{Tr}\left(\rho \cdot \lambda_{8}\right)=\frac{1}{2 \sqrt{3}}\left[\left\langle S_{x}^{2}\right\rangle+\left\langle S_{y}^{2}\right\rangle-2\left\langle S_{z}^{2}\right\rangle+3\left\langle S_{z}\right\rangle\right] .
$$

We can study particular cases with partial longitudinal and transversal polarizations. In the $\hat{z}$-axis basis we have $\rho_{\text {unpol }}=(1 / 3) \operatorname{diag}(1,1,1)$ and

$$
\rho_{\mathrm{pLP}}=\frac{1}{2}\left(\begin{array}{ccc}
H_{L}^{2} & 0 & 0 \\
0 & 2\left(1-H_{L}^{2}\right) & 0 \\
0 & 0 & H_{L}^{2}
\end{array}\right) ; \rho_{\mathrm{pTP}}=\frac{1}{4}\left(\begin{array}{ccc}
2-H_{T}^{2} & 0 & -2+3 H_{T}^{2} \\
0 & 2 H_{T}^{2} & 0 \\
-2+3 H_{T}^{2} & 0 & 2-H_{T}^{2}
\end{array}\right)
$$

where $H_{L}$ and $H_{T}$ are the fraction of longitudinally and transversally polarized hadrons, ${ }^{5}$ respectively. Here, $H^{2}=1$ represents the totally polarized case and $H^{2}=\frac{2}{3}$ describes an unpolarized hadron beam. In the former notation we identify

$$
\begin{aligned}
& \langle q q\rangle_{\mathrm{pLP}}=-3\left(\frac{2}{3}-H_{L}^{2}\right)\langle q q\rangle_{\mathrm{LP}}+3\left(1-H_{L}^{2}\right)\langle q q\rangle_{\mathrm{unpol}}, \\
& \langle q q\rangle_{\mathrm{pTP}}=-3\left(\frac{2}{3}-H_{T}^{2}\right)\langle q q\rangle_{\mathrm{TP}}+3\left(1-H_{T}^{2}\right)\langle q q\rangle_{\mathrm{unpol}},
\end{aligned}
$$

where the subindex $p$ stands for partial polarization. Then, the results for this part of the DIS differential cross sections corresponding to a partially polarized target are

$$
\frac{e^{4}}{16 \pi^{2} q^{4}} \text { y } l_{\mathrm{sym}}^{\mu \nu} W_{\mu \nu}^{\mathrm{Sb}}=\frac{M E e^{4}}{2^{2} \pi^{2} q^{4}}\left(\frac{2}{3}-H_{L}^{2}\right)\left[b_{1} x y^{2}+b_{2}(1-y)\right]+\mathcal{O}(\sqrt{-t}),
$$

for partial longitudinal polarization, and

$$
\frac{e^{4}}{16 \pi^{2} q^{4}} \text { y } l_{\mathrm{sym}}^{\mu \nu} W_{\mu \nu}^{\mathrm{Sb}}=\frac{M E e^{4}}{2^{3} \pi^{2} q^{4}}\left(H_{T}^{2}-\frac{2}{3}\right)\left[b_{1} x y^{2}+b_{2}(1-y)\right]+\mathcal{O}(\sqrt{-t}),
$$

for partial transversal polarization.

These expressions lead to the final results of DIS differential cross section for partially polarized hadrons as a generalization of the previous cases. For a partially longitudinally polarized target $H_{L}$ we obtain

$$
\begin{aligned}
\frac{d \sigma}{d x d y d \phi}=\frac{M E e^{4}}{4 \pi^{2} q^{4}}\{ & x y^{2}\left[F_{1}+\left(\frac{2}{3}-H_{L}^{2}\right) b_{1}\right]+(1-y)\left[F_{2}+\left(\frac{2}{3}-H_{L}^{2}\right) b_{2}\right] \\
& \left.-H_{l} H_{h}(2-y) y x g_{1}\right\},
\end{aligned}
$$

\footnotetext{
${ }^{5}$ Again, we are writing the transversal polarization along the $\hat{x}$-axis. However, by choosing the $\hat{y}$-axis instead it would lead to the same results.
} 
while for a partially transversally polarized target $H_{T}$ we obtain

$$
\begin{gathered}
\frac{d \sigma}{d x d y d \phi}=\frac{M E e^{4}}{4 \pi^{2} q^{4}}\left\{x y^{2}\left[F_{1}-\left(\frac{1}{3}-\frac{1}{2} H_{T}^{2}\right) b_{1}\right]+(1-y)\left[F_{2}-\left(\frac{1}{3}-\frac{1}{2} H_{T}^{2}\right) b_{2}\right]\right. \\
\left.+H_{l} H_{h} 2 x^{2} \sqrt{-t} \sqrt{1-y}\left(y g_{1}+2 g_{2}\right) \cos \phi\right\} .
\end{gathered}
$$

We have assumed longitudinally polarized leptonic beams $H_{l}$ in both cases. Once again, by assuming that $F_{2}(x)=2 x F_{1}(x)$ and $b_{2}(x)=2 x b_{1}(x)$ are satisfied, we recover the formulas shown in [11].

\subsection{Helicity amplitudes}

Deep inelastic scattering is related to the forward Compton scattering. Let us briefly describe the forward Compton scattering by using the virtual photon momentum $q$ and the hadron momentum $p$ in analogy with our DIS notation, and the variables $\lambda$ and $\lambda^{\prime}$ which denote the initial and final hadronic helicities. In the forward Compton scattering both the initial and final states of the system contain a photon and a hadron. The related amplitude is a function of a tensor $T_{\mu \nu}$ defined in terms of the matrix elements of two electromagnetic currents by the formula

$$
\left(T_{\mu \nu}\right)_{\lambda \lambda^{\prime}} \equiv i \int d^{4} x e^{i q \cdot x}\left\langle p, \lambda^{\prime}\left|T\left(J_{\mu}(x) J_{\nu}(0)\right)\right| p, \lambda\right\rangle
$$

which has a structure similar to the hadronic tensor $W^{\mu \nu}$ involved in DIS. On the other hand, the definition of $W_{\mu \nu}$ in terms of the currents is given by

$$
\left(W_{\mu \nu}\right)_{\lambda \lambda^{\prime}}=\frac{1}{4 \pi} \int d^{4} x e^{i q \cdot x}\left\langle p, \lambda^{\prime}\left|\left[J_{\mu}(x), J_{\nu}(0)\right]\right| p, \lambda\right\rangle .
$$

Since both $T_{\mu \nu}$ and $W_{\mu \nu}$ have the same Lorentz symmetry transformation properties, they have the same tensor structure decomposition. Thus, the information they contain can be encoded in their structure functions in the same way.

DIS and forward Compton scattering are related to each other by the optical theorem in quantum field theory as it is schematically shown in figure 2. It implies that twice the imaginary part of the forward Compton scattering amplitude leads to the total DIS amplitude. Therefore, the imaginary part of the $T_{\mu \nu}$ structure functions give the $W_{\mu \nu}$ structure functions times an extra numerical factor. Actually, this relation between the two processes is one of the basis of our construction to obtain the structure functions. As we will see in the next section, it is possible to describe a holographic dual version of the forward Compton scattering from the point of view of supergravity and string theory via the gauge/string duality.

The Compton amplitude can be analyzed for different helicities of the incoming and out-going particles. The amplitudes in each case are commonly denoted by $A_{h, H ; h^{\prime}, H^{\prime}}$, where the subindex $h$ corresponds to the helicity of the photon and $H$ labels the helicity of the hadron. Primed variables indicate helicities of final states, otherwise labels indicate helicities of initial states. These helicities can take three different values in the spin-one 


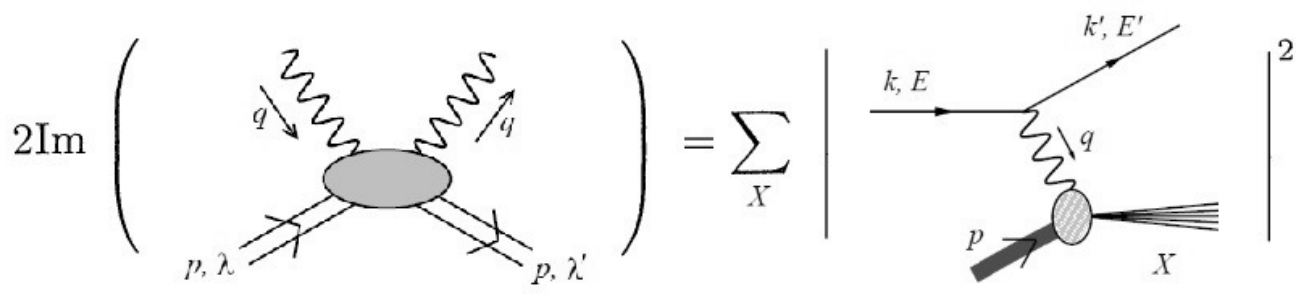

Figure 2. The relation between DIS and forward Compton scattering processes as given by the optical theorem in terms of Feynman diagrams.

case, $-1,+1$ and 0 , and they must satisfy the condition $h+H=h^{\prime}+H^{\prime}$. As shown in $[10,11]$ they can be calculated as

$$
A_{h H, h^{\prime} H^{\prime}}=\epsilon_{h}^{\mu \star} \epsilon_{h^{\prime}}^{\nu} T_{\mu \nu}\left(s_{H}\right)
$$

where the $\epsilon$ 's are the photon polarization vectors and $s_{H}$ is the hadron spin. For spinone hadrons there are eight independent helicity amplitudes. By considering a general kinematical regime, and by using the notation from [11], where $a_{3} \equiv b_{2} / 3-b_{3}$ and $a_{4} \equiv$ $b_{2} / 3-b_{4}$, these helicity amplitudes can be written in the following form

$$
\begin{aligned}
A_{++,++} & =F_{1}-\frac{\left(1-4 x^{2} t\right) b_{1}}{3}-\frac{x t}{3} a_{3}-g_{1}-4 x^{2} t g_{2} \\
& \simeq F_{1}-\frac{b_{1}}{3}-g_{1} \\
A_{+0,+0}= & F_{1}+\frac{2\left(1-4 x^{2} t\right) b_{1}}{3}+\frac{2 x t}{3} a_{3} \\
\simeq & F_{1}+\frac{2 b_{1}}{3} \\
A_{+0,0+}= & \sqrt{-t}\left[2 x\left(g_{1}+g_{2}\right)+\frac{a_{3}}{2}+\frac{\left(1-4 x^{2} t\right) a_{4}}{4}\right] \\
\simeq & \sqrt{-t}\left[2 x\left(g_{1}+g_{2}\right)+\frac{a_{3}}{2}+\frac{a_{4}}{4}\right] \\
A_{+-,+-} & =F_{1}-\frac{\left(1-4 x^{2} t\right) b_{1}}{3}-\frac{x t}{3} a_{3}+g_{1}+4 x^{2} t g_{2} \\
\simeq & F_{1}-\frac{b_{1}}{3}+g_{1}, \\
A_{+-, 00}= & \sqrt{-t}\left[2 x\left(g_{1}+g_{2}\right)-\frac{a_{3}}{2}-\frac{\left(1-4 x^{2} t\right) a_{4}}{4}\right] \\
\simeq & \sqrt{-t}\left[2 x\left(g_{1}+g_{2}\right)-\frac{a_{3}}{2}-\frac{a_{4}}{4}\right] \\
A_{+-,-+} & =-2 x t a_{3} \simeq 0, \\
A_{0+, 0+}= & -F_{1}+\frac{\left(1-4 x^{2} t\right) F_{2}}{2 x}+\frac{\left(1-4 x^{2} t\right) b_{1}}{3}-\frac{\left(3-12 x^{2} t+16 x^{4} t^{2}\right) b_{2}}{18 x} \\
& +\frac{4\left(-2 x^{2} t\right) b_{3}}{3 x}+\frac{2 x t}{3}\left(1-4 x^{2} t\right) b_{4} \\
\simeq & -F_{1}+\frac{F_{2}}{2 x}+\frac{b_{1}}{3}-\frac{b_{2}}{6 x}, \\
&
\end{aligned}
$$




$$
\begin{aligned}
A_{00,00}= & -F_{1}+\frac{\left(1-4 x^{2} t\right) F_{2}}{2 x}-\frac{2\left(1-4 x^{2} t\right) b_{1}}{3}+\frac{\left(3-12 x^{2} t+16 x^{4} t^{2}\right) b_{2}}{9 x} \\
& -\frac{8\left(-2 x^{2} t\right) b_{3}}{3 x}-\frac{4 x t}{3}\left(1-4 x^{2} t\right) b_{4} \\
\simeq & -F_{1}+\frac{F_{2}}{2 x}-\frac{2 b_{1}}{3}+\frac{b_{2}}{3 x} .
\end{aligned}
$$

Notice that the first line in each equation (3.36)-(3.43) coincides with the corresponding one in equations (7) in reference [11], ${ }^{6}$ and we write them here for completeness. On the other hand, the second line in each of these equations corresponds to the limit $|t| \ll 1$.

These amplitudes are useful tools in order to study the hadron structure, specially in the cases with $s>1 / 2$. They provide a simple way to understand the meaning of the structure functions in theoretical terms, and give important insights about how they can affect the scattering amplitudes and how to measure them. This is the reason why we shall explicitly show the form of these helicity amplitudes in terms of the structure functions we found by using the gauge/string duality.

For instance, in the spin-1/2 case one has $A_{+\uparrow,+\uparrow} \sim F_{1}-g_{1}$ and $A_{+\downarrow,+\downarrow} \sim F_{1}+$ $g_{1}$, which indicates that $F_{1}$ is the cross section for a transverse photon scattered off an unpolarized target, while $g_{1}$ is the spin asymmetry in the scattering cross section for a transverse photon [10]. One can also say that $g_{1}+g_{2}$ is proportional to the single helicity flip amplitude $\sim A_{+\downarrow, 0 \uparrow}$. For spin-one hadrons a similar analysis can be carried out. The function $F_{1} \sim A_{++,++}+A_{+0,+0}+A_{+-,+-}$can be interpreted in the same way. Also $b_{1} \sim A_{++,++}+A_{+-,+-}-2 A_{+0,+0}$ has an interpretation from a transverse photon scattered off a polarized spin-one target [11]. The combination $g_{1}+g_{2}$ also appears in the $A_{+-, 00}$ amplitude, and at first order in $t$ this is the only place where $g_{2}$ is present. The combinations $F_{1}-b_{1} / 3$ and $F_{2}-b_{2} / 3$ define $A_{0+, 0+}$ and will be important when we present our structure functions. Also, the $A_{+-,-+} \sim-b_{3}+b_{2} / 3$ is an interesting amplitude (even being subleading in $t$ ) since it characterizes the double helicity flip. As pointed out in $[10]^{7}$ this quantity does not receive contributions from quark interactions at first order in $\alpha_{s}$ because quarks cannot change their helicity by two. ${ }^{8}$

Finally, all these linear combinations of structure functions that define the helicity amplitudes are positive, and this is a consistency check for the structure functions we obtained in our previous papers [1-3] in terms of the holographic dual models.

\section{DIS differential cross sections from the gauge/string duality}

The present approach is based on a method developed by Polchinski and Strassler in [7], where the structure functions corresponding to glueball states were derived in terms of the gauge/string duality. They consider the planar limit of the $\mathcal{N}=1^{\star}$ SYM theory obtained from a deformation of the $\mathcal{N}=4 \mathrm{SYM}$ theory. The $\mathcal{N}=1^{\star} \mathrm{SYM}$ theory has

\footnotetext{
${ }^{6}$ This is easy to see by writing $\kappa$ in terms of $x$ and $t$, by taking into account an overall sign in the definition of the four-dimensional metric, and by noting that the definition of $\nu=P \cdot q$ in reference [11] differs from the one used in the present work which is $\nu=-P \cdot q / M$.

${ }^{7}$ In the mentioned paper this is the so-called $\Delta(x)$ structure function.

${ }^{8}$ Recall that $\alpha_{s}=g_{\mathrm{QCD}}^{2} / 4 \pi$.
} 
a running coupling and becomes conformal when the energies involved are higher than a color confinement scale $\Lambda$. Therefore, this SYM theory and QCD share some important qualitative properties: they exhibit confinement of the color degrees of freedom and a mass gap in the infrared. In the context of the gauge/string duality it was shown that the $\mathcal{N}=1^{\star}$ SYM theory is dual to strings propagating in a certain deformed $A d S_{5} \times S^{5}$ background [8]. In order to describe DIS, it has been calculated the scattering amplitude of a process where a particular graviton perturbation coming from the boundary couples to the dilaton, which is dual to a glueball state [7]. In addition, the optical theorem is used to relate DIS and forward Compton scattering. Therefore, in the gravity dual description the graviton and the dilaton perturbations are taken to describe both initial and final states leading to a process which turns out to be dual to the forward Compton scattering described in the previous section. ${ }^{9}$ It is important to emphasize that in the large- $x$ case the supergravity description is appropriate to derive the structure functions, while in the small- $x$ regime the string theory description is unavoidable.

The calculations of DIS from vector mesons have been presented in our papers [1-3]. We have obtained the leading order behavior of the eight structure functions involved in the hadronic tensor $W^{\mu \nu}$ for a spin-one target, namely $F_{1}, F_{2}, b_{1}, b_{2}, b_{3}, b_{4}, g_{1}$ and $g_{2}$ in terms of $x, \frac{\Lambda^{2}}{q^{2}}$ and some other parameters which define the holographic dual description.

The Bjorken parameter $x$ is a very important kinematic variable, and its physical range $0 \leq x \leq 1$ can be divided in four regions. ${ }^{10}$ In the region $1 / \sqrt{\lambda} \ll x<1$ (which we call the large- $x$ region or range A) the ten-dimensional Mandelstam variable associated with the intermediate state $\tilde{s} \sim\left(\alpha^{\prime 2} \lambda\right)^{-1 / 2}(1 / x-1)$ indicates that only massless string states are exchanged and thus we only have to consider the low energy limit of string theory, leading to a supergravity description. The second kinematic region (range B) is reached when $e^{-\sqrt{\lambda}} \ll x \ll 1 / \sqrt{\lambda}$. Thus, in this region the dynamics of string theory provides the appropriate description. Fortunately in this region, it is possible to think of locally interacting strings, in the sense that the characteristic length scales are smaller than the AdS radius, leading to some important simplifications [7]. This is the region we call small- $x$ (but not exponentially small). The third region (range $\mathrm{C}$ ) corresponding to $x \sim e^{-\sqrt{\lambda}}$ is important because the non-locality of the string scattering is described by a diffusion operator. This operator leads to the convergence of the first moments of the structure functions [7,9]. For smaller $x$ values, there is fourth region which is not accessible with the present approach since it would require to consider strings propagating in a curved background. Finally, we point out that for actual experiments large $x$ generally corresponds to $0.1<x<1$, while the small $x$ measurements are supposed to describe the $0<x<0.1$ region.

In the following sections we present our results for the structure functions in the first two regimes and show how they affect the differential cross section of the DIS process in the different polarization cases. We also consider the corresponding helicity amplitudes.

\footnotetext{
${ }^{9}$ For the large- $x$ case there is also a sum over intermediate dilaton states.

${ }^{10}$ Recall that $x=1$ corresponds to elastic scattering.
} 


\begin{tabular}{|c|c|c|c|}
\hline Model / Parameter & $\mathrm{p}$ & $\alpha$ & $\beta$ \\
\hline D3D7 & 7 & 2 & -2 \\
\hline D4D8 $\overline{\mathrm{D} 8}$ & 8 & $3 / 2$ & $-3 / 2$ \\
\hline D4D6 $\overline{\mathrm{D} 6}$ & 6 & $3 / 2$ & $-3 / 2$ \\
\hline
\end{tabular}

Table 1. Parameters for each Dp-brane model.

\subsection{Range A: $1 / \sqrt{\lambda} \ll x<1$}

The structure functions found in our previous paper [1] in this regime of the Bjorken parameter at leading order in $t$ are

$$
\begin{aligned}
F_{1} & =A^{\mathrm{SF}}(x) \frac{1}{12 x^{3}}(1-x), \\
F_{2} & =A^{\mathrm{SF}}(x) \frac{1}{6 x^{3}}(1-x), \\
b_{1} & =A^{\mathrm{SF}}(x) \frac{1}{4 x^{3}}(1-x), \\
b_{2} & =A^{\mathrm{SF}}(x) \frac{1}{2 x^{3}}(1-x), \\
b_{3} & =A^{\mathrm{SF}}(x) \frac{1}{24 x^{3}}(1-4 x), \\
b_{4} & =A^{\mathrm{SF}}(x) \frac{1}{12 x^{3}}(-1+4 x), \\
g_{1} & =A^{\mathrm{SF}}(x) \frac{t}{8 x^{2}}(-7+6 x), \\
g_{2} & =A^{\mathrm{SF}}(x) \frac{1}{16 x^{4}}(3-3 x),
\end{aligned}
$$

where

$$
A^{\mathrm{SF}}(x)=A_{0}^{\mathrm{SF}} \mu_{p}^{2} \mathcal{Q}^{2} \alpha^{\prime 4} R^{2 p-6}\left(\frac{\Lambda^{2}}{q^{2}}\right)^{\gamma} x^{\gamma+2 n+5}(1-x)^{\gamma-1},
$$

is the only model-dependent factor and it is written in terms of $q^{2}$ and the different variables involved in the string theory calculation: the Dp-brane tension $\mu_{p}$, the charge of the hadron $\mathcal{Q}$, the confining scale $\Lambda$, the sphere radius $R$, the string constant $\alpha^{\prime}$ and the exponents

$$
\gamma^{2}=\frac{A^{2}+\ell(\ell+p-5)}{B^{2}}, \quad A=\frac{1-2 \alpha-\beta(p-3) / 2-p+4}{2}, \quad B=\frac{\alpha-\beta-2}{2}
$$

related to the general induced asymptotic metric on the probe Dp-branes

$$
d s^{2}=\left(\frac{r}{R}\right)^{\alpha} \eta_{\mu \nu} d x^{\mu} d x^{\nu}+\left(\frac{r}{R}\right)^{\beta}\left[d r^{2}+r^{2} d \Omega_{p-4}^{2}\right] .
$$

Furthermore, we have defined $n=\frac{2+\beta}{4 B}$. Notice that $\ell$ is the usual spherical harmonic index that appears in the solutions of the scalar and vector mesons, and $A_{0}^{\mathrm{SF}}$ is a dimensionless normalization constant which depends on the model [1,2]. As explained in [2], for the three considered Dp-brane models the values of the parameters are shown in table 1. Using the 
redefinition $W_{i}=\frac{1}{12 x^{3}} A^{\mathrm{SF}}(x) \widetilde{W}_{i}$ we obtain

$$
\begin{aligned}
\widetilde{F_{1}} & =(1-x), & \widetilde{F_{2}} & =2(1-x), \\
\widetilde{b_{1}} & =3(1-x), & \widetilde{b_{2}} & =6(1-x) \\
\widetilde{b_{3}} & =\frac{1}{2}(1-4 x), & \widetilde{b_{4}} & =-(1-4 x), \\
\widetilde{g_{2}} & =\frac{3}{4 x}(3-3 x), & \widetilde{g_{1}} & =\frac{3 x t}{2}(-7+6 x) \approx 0 .
\end{aligned}
$$

This means that in this regime we obtain the relations $[1,2]$

$$
F_{2}=2 F_{1}, b_{2}=2 b_{1}, b_{1}=3 F_{1}, g_{2}=\frac{9}{4 x} F_{1}, b_{4}=-2 b_{3} .
$$

Note that the first two are typical Callan-Gross type relations, except for the lack of a factor $x$, which in this regime is understood as if the lepton is scattered by the entire hadron due to the strong coupling and the specific kinematic regime. By looking at the $q^{2}$-dependence we obtain a power-law decay, which is not exactly the same as in perturbative QCD. This was already observed in [7] where it was pointed out that at weak 't Hooft coupling the leading contribution comes from twist-2 operators, while at strong coupling the dominant contribution is given by double-trace operators.

For completeness we also write the results for the scalar mesons, which as mentioned only have two structure functions

$$
F_{1}=0, F_{2}=A_{0}^{\text {scalar }} \mu_{p}^{2} \mathcal{Q}^{2} \alpha^{\prime 4} R^{2 p-6}\left(\frac{\Lambda^{2}}{q^{2}}\right)^{\gamma+1} x^{\gamma+3+2 n}(1-x)^{\gamma},
$$

where $A_{0}^{\text {scalar }}=2^{2 \gamma+4} B^{2 \gamma+2} \pi^{5}\left|c_{i}\right|^{2}\left|c_{X}\right|^{2} \frac{\Gamma^{2}[\gamma+n+2]}{\Gamma^{2}[n+1]}$, while the constants $c_{i}$ and $c_{X}$ have been defined in [1].

By plugging the structure functions from the holographic vector mesons in the general spin-one DIS differential cross section we find

$$
\begin{aligned}
\frac{d \sigma}{d x d y d \phi}= & \frac{M E e^{4}}{4 \pi^{2} q^{4}}\left\{\langle q q\rangle\left[x y^{2}+2(1-y)\right] F_{1}+2 x y^{2} g_{2}\left[\frac{\left(s_{h} \cdot s_{l}\right)}{(P \cdot q)}-\frac{\left(q \cdot s_{h}\right)\left(P \cdot s_{l}\right)}{(P \cdot q)^{2}}\right]\right\} \\
= & \frac{M E e^{4}}{4 \pi^{2} q^{4}} F_{1}\left\{\langle q q\rangle\left[x y^{2}+2(1-y)\right]+\frac{9}{2} y^{2}\left[\frac{\left(s_{h} \cdot s_{l}\right)}{(P \cdot q)}-\frac{\left(q \cdot s_{h}\right)\left(P \cdot s_{l}\right)}{(P \cdot q)^{2}}\right]\right\} \\
= & \frac{M E e^{4}}{4 \pi^{2} q^{4}} \frac{A^{\mathrm{SF}}(x)(1-x)}{12 x^{3}} \times \\
& \left\{\langle q q\rangle\left[x y^{2}+2(1-y)\right]+\frac{9}{2} y^{2}\left[\frac{\left(s_{h} \cdot s_{l}\right)}{(P \cdot q)}-\frac{\left(q \cdot s_{h}\right)\left(P \cdot s_{l}\right)}{(P \cdot q)^{2}}\right]\right\} .
\end{aligned}
$$

For a longitudinally polarized lepton beam where $s_{l}=H_{l} k$ with $H_{l}= \pm 1$ for positive and negative helicity, we can rewrite this expression in the following form

$$
\frac{d \sigma}{d x d y d \phi}=\frac{M E e^{4} A^{\mathrm{SF}}(x)}{48 \pi^{2} q^{4} x^{3}}(1-x)\left\{\langle q q\rangle\left[x y^{2}+2(1-y)\right]-\frac{9 H_{l}}{2 M E} s_{h}^{\mu}\left(y k_{\mu}-q_{\mu}\right)\right\} .
$$

We can study the behavior of this result for each polarization as follows. 
- Unpolarized target: $\langle q q\rangle_{\mathrm{unpol}}=1, s_{h}=0$.

$$
\left.\frac{d \sigma}{d x d y d \phi}\right|_{\text {unpol }}=\frac{M E e^{4} A^{\mathrm{SF}}(x)}{48 \pi^{2} q^{4} x^{3}}(1-x)\left[x y^{2}+2(1-y)\right] .
$$

- Longitudinally polarized target: $\langle q q\rangle_{\mathrm{LP}}=0, s_{h}=\left(0, H_{h} M \hat{z}\right)$ (and using $\cos \theta \approx 1$ ).

$$
\left.\frac{d \sigma}{d x d y d \phi}\right|_{\mathrm{LP}}=0
$$

This is the result at leading order. It is easy to see why this happens: since $\langle q q\rangle_{\mathrm{LP}}=0$, the $b_{i}=3 F_{i}$ relations for $i=1,2$ imply that the $b-F$ part of the cross section vanishes. Now, as pointed out in [10] $s_{h}$ is dotted with $k$ or $q$ both of which have similar 0 and 3 components at leading order. Thus, we replace it by $-H_{h} p^{11}$ without changing the results. This implies that the $g_{2}$-piece becomes sub-leading in this case, and as $g_{1} \approx 0$ at first order, therefore the cross section vanishes.

- Transversally polarized target: $\langle q q\rangle_{\mathrm{TP}}=3 / 2, s_{h}=\left(0, H_{h} M \hat{x}\right)$

$$
\left.\frac{d \sigma}{d x d y d \phi}\right|_{\mathrm{TP}}=\frac{M E e^{4} A^{\mathrm{SF}}(x)}{48 \pi^{2} q^{4} x^{3}}(1-x)\left\{\frac{3}{2}\left[x y^{2}+2(1-y)\right]-\frac{9 H_{l} H_{h}}{2} \frac{M}{|q|}(1-y) \cos \phi\right\}
$$

Note that the $M /|q|=\sqrt{-t}$ factor makes the spin-dependent term sub-leading. The reader could think that it is not a reliable result since in early steps of our calculations we have ignored this kind of terms. This is not the case because this has been done in terms involving the $b_{i}$ functions, which contribute to the leading term, and mainly because the spin-dependent part is easily distinguished within experiments.

In addition, for the helicity amplitudes in this case we obtain

$$
\begin{aligned}
A_{++,++} & \simeq 0+O(t), \\
A_{+0,+0} & \simeq 3 F_{1} \\
A_{+0,0+} & \simeq \sqrt{-t}\left[\frac{9 F_{1}}{2}+\frac{a_{3}}{2}+\frac{a_{4}}{4}\right]=\sqrt{-t} 6 F_{1}, \\
A_{+-,+-} & \simeq 0+O(t), \\
A_{+-, 00} & \simeq \sqrt{-t}\left[\frac{9 F_{1}}{2}-\frac{a_{3}}{2}-\frac{a_{4}}{4}\right]=\sqrt{-t} 3 F_{1}, \\
A_{+-,-+} & \simeq 0 \\
A_{0+, 0+} & \simeq \frac{4 b_{3}}{3 x}, \\
A_{00,00} & \simeq 3\left(\frac{1}{x}-1\right) F_{1}-\frac{8 b_{3}}{3 x} .
\end{aligned}
$$

\footnotetext{
${ }^{11}$ The minus sign accounts for the difference in the four-dimensional Minkowski metric components $\eta_{00}$ and $\eta_{z z}$.
} 


\subsection{Range B: $e^{-\sqrt{\lambda}} \ll x \ll 1 / \sqrt{\lambda}$}

The results we have obtained in our previous paper [3] in the small- $x$ (but not exponentially small) regime for the polarized vector meson structure functions can be summarized in the following equations

$$
\begin{aligned}
F_{1} & =\frac{1}{12 x^{2}} I_{1}, & F_{2} & =\frac{1}{6 x}\left(I_{1}+I_{0}\right), \\
b_{1} & =\frac{1}{4 x^{2}} I_{1}, & b_{2} & =\frac{1}{2 x}\left(I_{1}+I_{0}\right), \\
b_{3} & =-\frac{1}{3 x}\left(I_{1}+I_{0}\right), & b_{4} & =\frac{1}{6 x}\left(I_{1}+I_{0}\right), \\
g_{1} & =0, & g_{2} & =\frac{1}{8 x^{2}}\left(I_{1}+I_{0}\right),
\end{aligned}
$$

where $I_{1}$ and $I_{0}$ are $x$-independent factors given by integrals of Bessel functions and some constants, plus the $q^{2}$-dependence. According to these results, in this regime the CallanGross type relations become

$$
F_{2}=2 x F_{1}\left(1+\frac{I_{0}}{I_{1}}\right), \quad b_{2}=2 x b_{1}\left(1+\frac{I_{0}}{I_{1}}\right), \quad b_{4}=\frac{1}{3} b_{2}=-\frac{1}{2} b_{3} .
$$

Notice that the first two relations have a Bjorken parameter factor $x$ with respect to the ones in the regime A described in the previous section. The pre-factors are given by

$$
\begin{aligned}
& I_{1}=C \frac{\alpha^{2} \Sigma^{2} q^{4}}{\sqrt{\lambda} \Lambda^{4} \Gamma^{2}(n+1) B}\left(\frac{q R}{B}\right)^{(2 n+1)-D} I_{n+1, D}, \\
& I_{0}=C \frac{\alpha^{2} B \Sigma^{2} q^{2}}{\sqrt{\lambda} \Lambda^{4} R^{2} \Gamma^{2}(n+1)}\left(\frac{q R}{B}\right)^{(2 n+3)-D} I_{n, D},
\end{aligned}
$$

where $C$ is a normalization constant and $D=2 n+1+\frac{1}{B}\left[2 \Delta+\alpha+\left(\frac{\beta}{2}+1\right)(p-5)\right]$. We have used the definition $\Sigma^{2}=\Delta^{2}+\Delta(\Delta-2)$. This is where the $\ell$-dependence enters, since $\Delta=\gamma(\ell) B-A$. Note that the $x$-dependence of the structure functions is the same for all models, which was not the case in the previous section. The Bessel function integrals are defined as ${ }^{12}$

$$
\int_{0}^{\infty} d \omega \omega^{m} K_{n}^{2}(\omega)=I_{n, m}=2^{m-2} \frac{\Gamma(\nu+n) \Gamma(\nu-n) \Gamma^{2}(\nu)}{\Gamma(2 \nu)}, \quad \nu=\frac{1}{2}(m+1) .
$$

In the $\mathrm{D} 4 \mathrm{D} 8 \overline{\mathrm{D} 8}$-brane and $\mathrm{D} 4 \mathrm{D} 6 \overline{\mathrm{D} 6}$-brane models $K_{n}(w)$ functions turn out to be fractional Bessel functions, however all the integrals are finite. Again, all these factors are independent of $x$ and $y .{ }^{13}$ Finally, the only model-dependent and $\ell$-dependent numerical factor that enters the Callan-Gross type relations is

$$
\delta \equiv 1+\frac{I_{0}}{I_{1}}=1+\frac{I_{n, D}}{I_{n+1, D}}=\frac{2 D}{D+2 n+1} .
$$

\footnotetext{
${ }^{12}$ Recall that this result is valid provided that the arguments of the gamma functions are positive.

${ }^{13}$ We refer the reader to [3] for a careful derivation and analysis of these results.
} 


\begin{tabular}{|c|c|c|c|}
\hline Model & D3D7 & D4D8 $\overline{\mathrm{D} 8}$ & D4D6 $\overline{\mathrm{D} 6}$ \\
\hline$\delta$ & $\frac{2 \Delta+3}{\Delta+2}$ & $\frac{4(4 \Delta+3)}{8 \Delta+9}$ & $\frac{16(\Delta+1)}{8 \Delta+11}$ \\
\hline
\end{tabular}

Table 2. Parameter $\delta$ in each model.

Table 2 shows the values of $\delta$ for the three models we consider. In all the cases we have $1<\delta<2$ since $\Delta$ is positive. As for the scalar mesons the results are the following

$$
\begin{aligned}
& F_{1}=\frac{\pi^{2}}{16 x^{2}} \rho_{3}\left|c_{i}\right|^{2}\left(\frac{\Lambda^{2}}{q^{2}}\right)^{\Delta-1} \frac{1}{\sqrt{4 \pi g_{c} N}} I_{1,2 \Delta+3}, \\
& F_{2}=\frac{\pi^{2}}{8 x} \rho_{3}\left|c_{i}\right|^{2}\left(\frac{\Lambda^{2}}{q^{2}}\right)^{\Delta-1} \frac{1}{\sqrt{4 \pi g_{c} N}}\left(I_{0,2 \Delta+3}+I_{1,2 \Delta+3}\right) .
\end{aligned}
$$

Equations (4.14) and (4.15) show the dependence of our small- $x$ structure functions with the momentum of the virtual photon: they always fall-off as a power of $q^{2}$. In the Regge regime the calculations and measurements suggest that a Pomeron is exchanged ${ }^{14}$ both at weak and strong 't Hooft coupling. However, there is an important difference: when $\lambda$ is small the virtual photon scatters off a parton inside the hadron, leading to a small (growing) dependence in $q^{2}$, while at large $\lambda$ a Pomeron is supposed to collide with the entire hadron. This is what happens in the present case, since we are using the gauge/string duality at strong coupling. Thus, we have obtained the same $q^{2}$-power fall-off as in the large- $x$ case, and the amplitude is again dominated by the same double-trace operators [7].

Plugging our structure functions in the most general DIS differential cross section for a spin-one target we obtain

$$
\begin{aligned}
\frac{d \sigma}{d x d y d \phi} & =\frac{M E e^{4}}{4 \pi^{2} q^{4}}\left\{\langle q q\rangle\left[x y^{2}+2 x(1-y) \delta\right] F_{1}+2 x y^{2} g_{2}\left[\frac{\left(s_{h} \cdot s_{l}\right)}{(p \cdot q)}-\frac{\left(q \cdot s_{h}\right)\left(p \cdot s_{l}\right)}{(p \cdot q)^{2}}\right]\right\} \\
& =\frac{M E e^{4}}{4 \pi^{2} q^{4}} F_{1}\left\{\langle q q\rangle\left[x y^{2}+2 x(1-y) \delta\right]+3 x y^{2} \delta\left[\frac{\left(s_{h} \cdot s_{l}\right)}{(p \cdot q)}-\frac{\left(q \cdot s_{h}\right)\left(p \cdot s_{l}\right)}{(p \cdot q)^{2}}\right]\right\} \\
& =\frac{M E e^{4}}{48 \pi^{2} q^{4}} \frac{I_{1}}{x}\left\{\langle q q\rangle\left[y^{2}+2(1-y) \delta\right]+3 y^{2} \delta\left[\frac{\left(s_{h} \cdot s_{l}\right)}{(p \cdot q)}-\frac{\left(q \cdot s_{h}\right)\left(p \cdot s_{l}\right)}{(p \cdot q)^{2}}\right]\right\} .
\end{aligned}
$$

Once again, the experimentally relevant situation occurs when the lepton is longitudinally polarized, i.e. when $s_{l}=H_{l} k$ with $H_{l}= \pm 1$. Therefore, the differential cross section takes the form

$$
\frac{d \sigma}{d x d y d \phi}=\frac{M E e^{4}}{48 \pi^{2} q^{4}} \frac{I_{1}}{x}\left\{\langle q q\rangle\left[y^{2}+2(1-y) \delta\right]-\frac{3 \delta}{M E} H_{l} s_{h}^{\mu}\left(y k_{\mu}-q_{\mu}\right)\right\} .
$$

Then, we can study the behavior of this result for each hadronic polarization as follows.

- Unpolarized target: $\langle q q\rangle_{\text {unpol }}=1, s_{h}=0$. In this case the spin-dependent term vanishes automatically and we obtain

$$
\left.\frac{d \sigma}{d x d y d \phi}\right|_{\text {unpol }}=\frac{M E e^{4}}{48 \pi^{2} q^{4}} \frac{I_{1}}{x}\left[y^{2}+2(1-y) \delta\right] .
$$

\footnotetext{
${ }^{14}$ There are some important differences between hard and soft Pomerons that we shall not address here, for this see [9].
} 
- Longitudinally polarized target: $\langle q q\rangle_{\mathrm{LP}}=0, s_{h}=\left(0, H_{h} M \hat{z}\right)$

$$
\left.\frac{d \sigma}{d x d y d \phi}\right|_{\mathrm{LP}}=0 \text {. }
$$

This is just like in the large- $x$ regime. We obtain a vanishing result at leading order since $g_{1}=0$ (notice that the $g_{1}$ structure function together with the difference $F_{1}-\frac{1}{3} b_{1}$ give the main contribution to the longitudinally polarized cross section), and this is a consistency check of our results at small $x$ for the polarized vector meson structure functions.

- Transversally polarized target: $\langle q q\rangle_{\mathrm{TP}}=3 / 2, s_{h}=M H_{h} \hat{x}$. Now, we have a vanishing $s_{h} \cdot k$ but a non-vanishing $s \cdot q=M H_{h} k^{\prime x}=M E^{\prime} H_{h} \sin \theta \cos \phi$, and since $E^{\prime}=E(1-y)$ and $\sin (\theta) \sim \theta \sim M /|q|$ we finally obtain

$$
\left.\frac{d \sigma}{d x d y d \phi}\right|_{\mathrm{TP}}=\frac{M E e^{4}}{48 \pi^{2} q^{4}} \frac{I_{1}}{x}\left\{\frac{3}{2}\left[y^{2}+2(1-y) \delta\right]-3 \delta(1-y) \frac{M}{|q|} H_{l} H_{h} \cos \phi\right\} .
$$

Since $M /|q| \sim \sqrt{-t}$ the second term also becomes sub-leading in this case.

We can also obtain the helicity amplitudes in this regime which become

$$
\begin{aligned}
A_{++,++} & \simeq 0, & A_{+0,+0} & \simeq 3 F_{1}, \\
A_{+0,0+} & \simeq 0, & A_{+-,+-} & \simeq 0, \\
A_{+-, 00}=O(\sqrt{-t}) & \approx 0, & A_{+-,-+} & =0, \\
A_{0+, 0+}=2(\delta-1) F_{1} & >0, & A_{00,00} & =3(\delta-1) F_{1}>0 .
\end{aligned}
$$

Note that in this parametric region the factor $a_{3}=b_{2} / 3-b_{3}$ defined in the section 3.3 becomes $a_{3}=b_{2}$ at leading order. This is interesting since it means that the $b_{2}$ structure function characterizes the double helicity flip amplitude. The corresponding $A_{+-,-+}$helicity amplitude is proportional to $t$, thus it is always small in the DIS regime, but the $b_{2} \sim x^{-1}$ growth could in principle lead to a non-vanishing helicity amplitude.

\subsection{Range C: $x \sim e^{-\sqrt{\lambda}}$}

This is the exponentially small region of the Bjorken parameter. In the region B we have neglected the factor $\tilde{s}^{\alpha^{\prime}} \tilde{t} / 2$. The effect of this factor is very important within the parametric region $x \sim \exp (-\sqrt{\lambda})[3]$. The tildes indicate ten-dimensional Mandelstam variables. Let us consider the strong coupling regime $1 \ll \lambda \ll N,{ }^{15}$ and exponentially large values of $\tilde{s}$ with $\frac{\log s}{\sqrt{\lambda}}$ fixed. In this region the interaction cannot be considered local in the AdS background, since $\tilde{t}$ becomes a differential operator $[7,9]$, and consequently we have to replace it by the following operator

$$
\alpha^{\prime} \tilde{t} \rightarrow \alpha^{\prime} \nabla^{2}=\alpha^{\prime}\left(\frac{R^{2} t}{r^{2}}+\nabla_{\perp}^{2}\right)
$$

\footnotetext{
${ }^{15}$ In this subsection we focus on the D3D7-brane model, the other models have a few modifications but the discussion is very similar.
} 
which takes into account the momentum transfer in the transverse directions, and it induces the factor

$$
\left(\alpha^{\prime} \tilde{s}\right)^{\alpha^{\prime} \tilde{t} / 2} \sim x^{-\alpha^{\prime} \tilde{t} / 2} \sim x^{-\alpha^{\prime} \nabla^{2} / 2} .
$$

The physical meaning of this is that within this region $\mathrm{C}$ there is a Pomeron exchange in the $t$-channel. ${ }^{16}$ The consequence of this leads to include a factor $x^{\alpha^{\prime} \zeta / 2}$ multiplying the structure functions. This is obtained in the region $\mathrm{C}$ by replacing the differential operator $-\nabla^{2}=-D^{2}$ by its smallest eigenvalue $\zeta$. Then, the structure functions become $F_{1} \propto x^{-2+\alpha^{\prime} \zeta / 2}$ and $F_{2} \propto x^{-1+\alpha^{\prime} \zeta / 2}$, where the Pomeron intercept is identified as $2-\alpha^{\prime} \zeta / 2$. This is the expected behavior for these functions. In particular, for the D3D7-brane model we have $\zeta=\frac{4}{R^{2}}\left(1-\Delta^{2}\right) \leq 0$. This implies that in our results of the previous section 4.2 in the region $\mathrm{B}$ we have to multiply all the structure functions by the factor $x^{\alpha^{\prime} \zeta / 2}$. This implies that the differential cross sections for different meson polarizations as well as the helicity amplitudes must also be multiplied by this factor in the parametric region $\mathrm{C}$.

\section{Results and discussion}

In this section we present our results and discuss them.

\subsection{Structure functions and differential cross sections}

Let us begin with the case of vector mesons. Figures 3, 4 and 5 show the structure functions $F_{1}, F_{2}, b_{1}$ and $b_{2}$ for polarized vector mesons as a function of the Bjorken parameter. We consider the D3D7-, D4D8 $\overline{\mathrm{D} 8}$ - and D4D6 $\overline{\mathrm{D} 6}$-brane models, respectively. In each case we focus on the lightest particles given by $\ell=1,2,3$. For the small- $x$ region the structure functions $F_{1}, F_{2}, b_{1}$ and $b_{2}$ are represented by a continuously decreasing curve, which has very little dependence on the values of $\ell=1,2,3$. On the other hand, for large $x$-values all the figures show a set of bell-shaped curves with maxima around $x \simeq 0$.6. The height of these curves decreases as $\ell$ increases. The normalization constants are specified in the next section and, they have been chosen in order to have the best fitting to the lattice QCD results of the first three moments of the $\rho$ meson. ${ }^{17}$ These moments are shown in table 5 and discussed below. Recall that in the small- $x$ parameter region there is a model-dependent and $\ell$-dependent factor in the structure functions. All the structure functions behave similarly. On the one hand, the $x>0.2$ region is dominated by a smooth bell-shaped function with maximum around $x \simeq 0.6$ (the specific value depends on $\ell$, as it can be seen from equations (4.1) and (4.2)). It approaches zero as $x \rightarrow 1$. On the other hand, as $x$ decreases these bell-shaped functions also decrease, and the small- $x$ structure functions defined in equations (4.12), (4.14) and (4.15) dominate. The $x<0.1$ region is described by functions $x^{-1}$ or $x^{-2}$ (depending on which structure function one considers). The two type of functions (the hyperbolic and the bell-shaped ones) overlap for $0.1<x<0.2$, which is a transition region between both regimes of the Bjorken parameter. This behavior is understood from the Callan-Gross type relations in each range of the

\footnotetext{
${ }^{16}$ In reference [3] we have not considered multi-Pomeron exchange.

${ }^{17} \mathrm{We}$ omit the possible dependence of these constants with $\ell$ since we compare with the moments of the structure functions for $\ell=1$, i.e. the moments corresponding to the $\rho$ meson, so the curves for $\ell>1$ have been drawn for the same values of the constants as for $\ell=1$.
} 


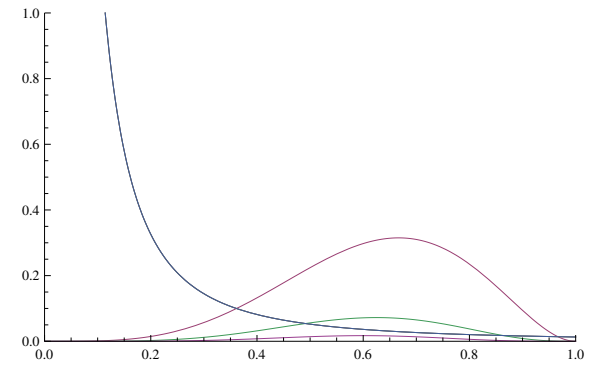

(a) $F_{1}(x)$ function.

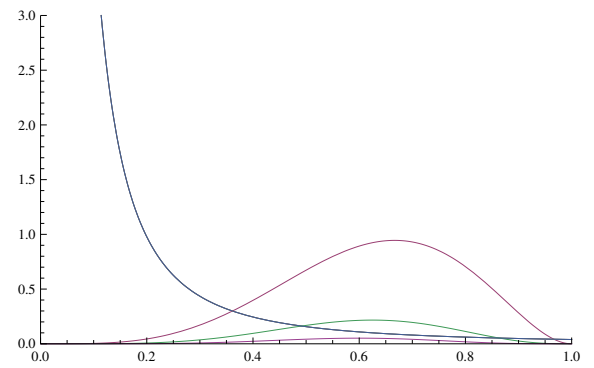

(c) $b_{1}(x)$ function.

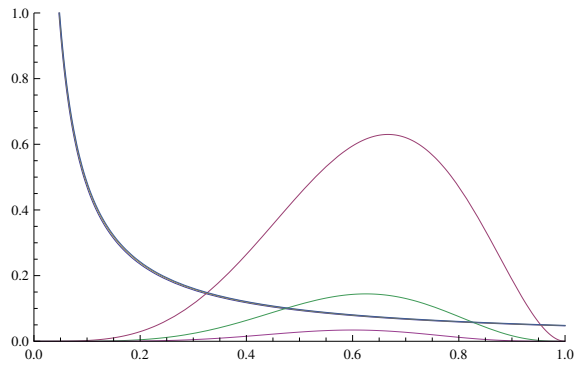

(b) $F_{2}(x)$ function.

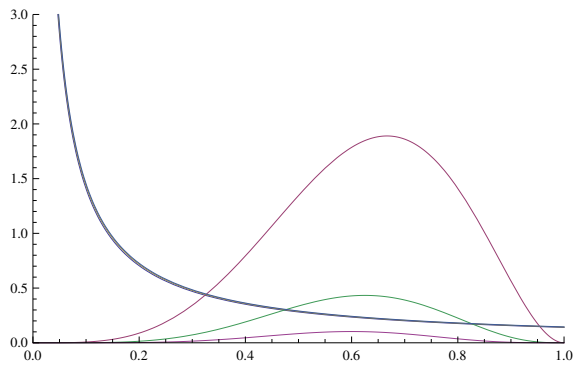

(d) $b_{2}(x)$ function.

Figure 3. Structure functions $F_{1}, F_{2}, b_{1}$ and $b_{2}$ in terms of the Bjorken parameter $x$ for the $\ell=1,2$ and 3 vector mesons in the D3D7-brane model. Bell-shaped curves correspond to the large- $x$ region (height decreases as $\ell$ increases) while the hyperbolic curves correspond to the small- $x$ region.

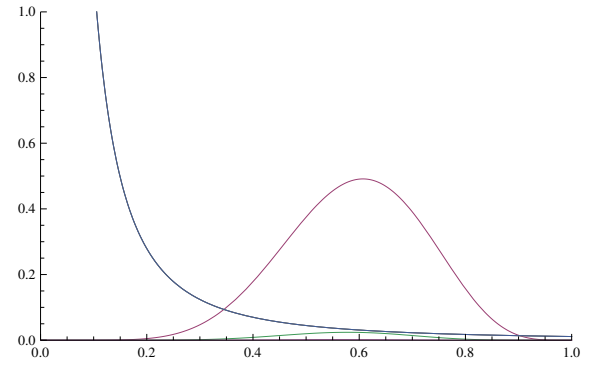

(a) $F_{1}(x)$ function.

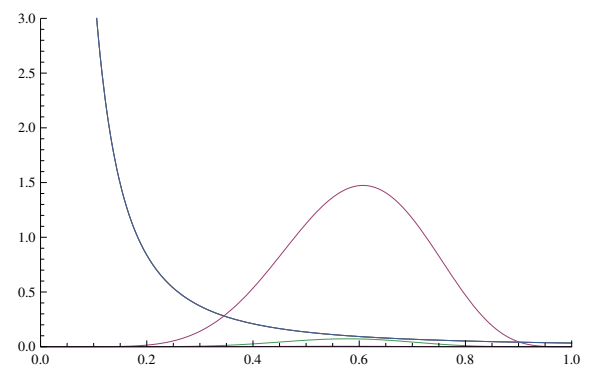

(c) $b_{1}(x)$ function.

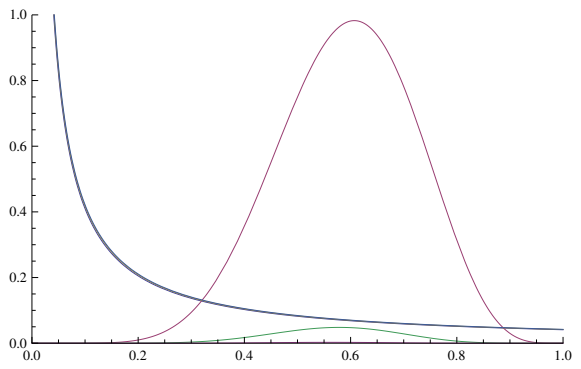

(b) $F_{2}(x)$ function.

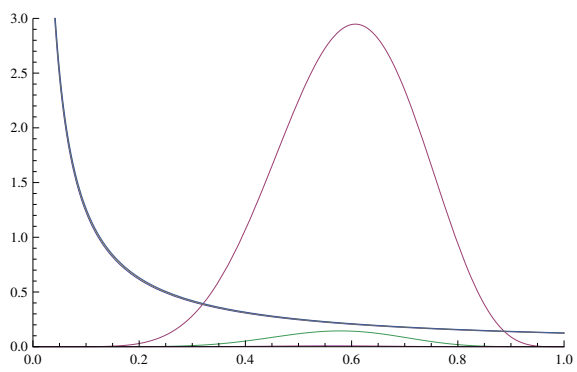

(d) $b_{2}(x)$ function.

Figure 4. Structure functions $F_{1}, F_{2}, b_{1}$ and $b_{2}$ in terms of the Bjorken parameter $x$ for the $\ell=1,2$ and 3 vector mesons in the D4D8 $\overline{\mathrm{D} 8}$-brane model. Bell-shaped curves correspond to the large- $x$ region (height decreases as $\ell$ increases) while the hyperbolic curves correspond to the small- $x$ region. 


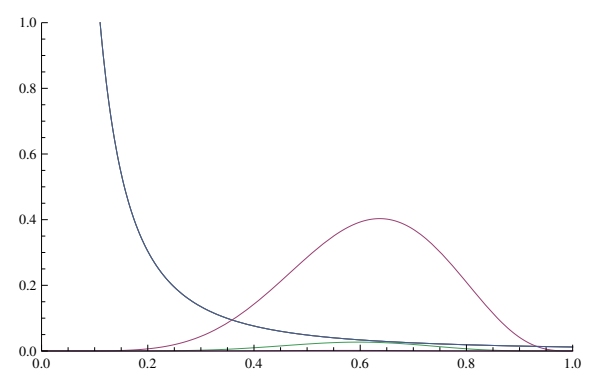

(a) $F_{1}(x)$ function.

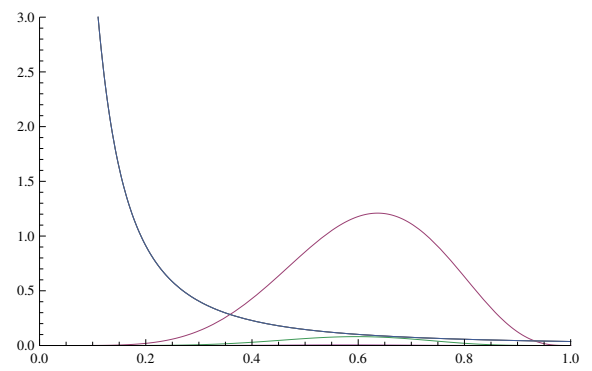

(c) $b_{1}(x)$ function.

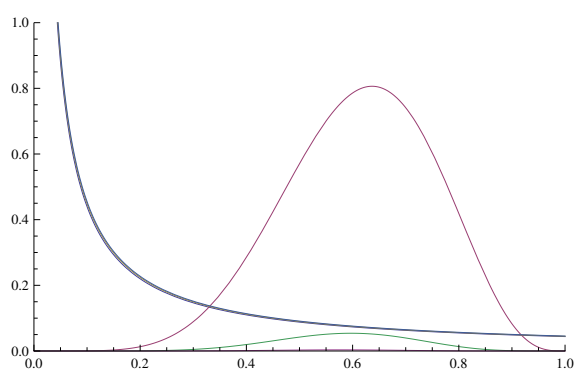

(b) $F_{2}(x)$ function.

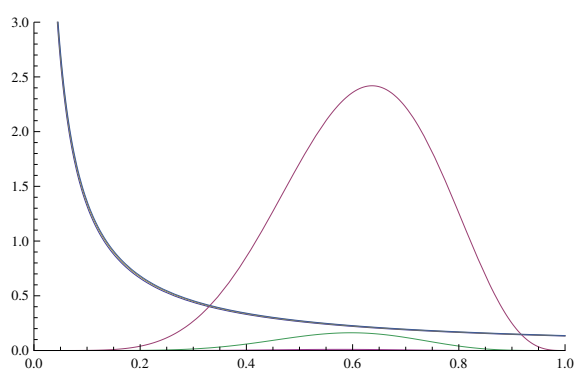

(d) $b_{2}(x)$ function.

Figure 5. Structure functions $F_{1}, F_{2}, b_{1}$ and $b_{2}$ in terms of the Bjorken parameter $x$ for the $\ell=1,2$ and 3 vector mesons in the D4D6 $\overline{\mathrm{D} 6}$-brane model. Bell-shaped curves correspond to the large- $x$ region (height decreases as $\ell$ increases) while the hyperbolic curves correspond to the small- $x$ region.

Bjorken parameter, as well as from the fact that $b_{i}=3 F_{i}$ in both cases. Also, remember that when $x$ becomes exponentially small this description fails because the approximation that strings interact locally enough in order to use a flat space amplitudes breaks down. In this case the correct behavior should be obtained by considering a diffusion operator, and ultimately the description of full string theory on a curved background. The analysis in this parameter region is very difficult. However, there is evidence that the divergence disappears (at least for the glueball DIS [7]), leading to finite moments of the structure functions, but this is beyond the scope of this work. In the next section we will deal with this by introducing a low- $x$ cutoff. We can see that for $\ell=1$ it gives similar curves for the three models we consider, even if the normalization constants are different for each model. It is interesting to see that the structure functions at small $x$ have similar behavior for $\ell=1,2,3$, while at large $x$ they get smaller as the parameter $\ell$ increases. This is more evident in the D4D8 $\overline{\mathrm{D} 8}$ and D4D6 $\overline{\mathrm{D} 6}$-brane models than in the D3D7-brane model. In those cases, for $\ell=3$ the corresponding curve is one order of magnitude smaller than the corresponding one for $\ell=1$ of the large- $x$ structure function.

We can also compare with the existing literature. In particular, at large $x$ the curves for $F_{1}$ and $F_{2}$ have a similar bell-shaped behavior as those presented in [12], although the maxima in that reference occurs for $x \simeq 0.8$. In [12] the DIS structure functions for the lightest vector mesons (they only consider the unpolarized case) have been obtained by using the $\mathrm{D} 4 \mathrm{D} 8 \overline{\mathrm{D}} 8$-brane model, i.e. the $\rho$ meson and the $a_{1}$ axial-vector meson. In 
fact, in that reference $F_{1}$ and $F_{2}$ structure functions have been obtained in the range $0.2<x<1$. We recall that the calculations in that reference are different from ours since they considered a four-dimensional effective Lagrangian only describing the $1 / \sqrt{\lambda} \ll x<1$ range. Obviously, this describes only a part of the problem. Instead, in [1, 2] we have derived the corresponding Lagrangians for the three holographic dual models from first principles from type IIA and type IIB supergravities within this parametric region, while for small $x$ in our paper [3] we have obtained the structure functions by using type IIA and type IIB string theories. From the structure functions obtained in [12] they found the ratio $F_{2} /\left(2 F_{1}\right) \approx 1$, satisfying this Callan-Gross type relation (as expected from the supergravity calculation [7] within this parametric range, note that it has not the factor $x$ multiplying $F_{1}$ which agrees with our results) within the interval $0.4<x<0.6$ and momentum transfer $10 \mathrm{GeV}^{2}<\left|q^{2}\right|<80 \mathrm{GeV}^{2}$. However, in [1-3] we have proved that a modified version of the Callan-Gross relation holds within the whole range of the Bjorken parameter and for any value of the momentum transfer, in fact there appear two Callan-Gross type relations: $F_{2}=2 F_{1}$ for $1 / \sqrt{\lambda} \ll x<1$ (supergravity) and $F_{2}=2 x F_{1} \delta$ for $\exp (-\sqrt{\lambda}) \ll x \ll 1 / \sqrt{\lambda}$ (from superstring theory, see section 4.2). Note that $0.5<\delta<1$ for all mesons described by the models we consider. Moreover, we have also obtained new exact relations among the structure functions for scalar and for polarized vector mesons in the planar limit at strong 't Hooft coupling. In addition, in reference [12] only structure functions for the lowest states of vector and axial vector mesons were studied, while our results hold for any scalar or polarized vector meson.

Now, we present our results for the DIS differential cross section of unpolarized vector mesons depicted as contour line maps in terms of $x$ and $y$ variables. We show that for large $x$ in figure 6 and for small $x$ in figure 7, and for the three different Dp-brane models we have considered. Broader curves in figure 6 correspond to lower values of the DIS differential cross section. On the other hand, in figure 7 curves with larger slope correspond to larger values of the DIS differential cross section. In figure 6 we can observe that the differential cross section of unpolarized holographic vector mesons is larger around $x \approx 0.5$, and also it becomes less spread for the Sakai-Sugimoto model. Another feature is that this differential cross section is larger for smaller values of the fractional energy loss of the charged lepton $y$, having a maximum when the energy of the incident lepton and the one of the scattered lepton are the same. On the other hand, curves in figure 7 show contour line maps of DIS differential cross section for unpolarized holographic vector mesons for the three flavor-brane models we describe, in the region $\exp (-\sqrt{\lambda}) \ll x \ll 1 / \sqrt{\lambda}$. The horizontal axis represents the Bjorken parameter within the range $[0.01,0.1]$, while the vertical one corresponds to the variable $y,[0,1]$.

Next, let us focus on the comparison with phenomenology of the meson structure functions. This turns out to be difficult because the experimental data of these functions is actually much more limited than in the case of baryons. Moreover, it is more difficult to carry out such a comparison when the Bjorken parameter $x$ is small. In the case of pions there have been important phenomenological studies [13-16], however all these investigations focus on the valence structure function related to fixed-target pion DrellYan experiments. The corresponding data are restricted to the parametric region $x \geq 0.2$. 


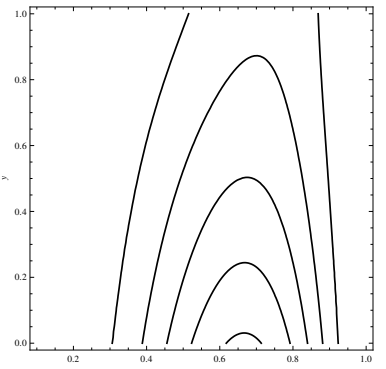

(a) $\mathrm{D} 3 \mathrm{D} 7 \& \ell=1$

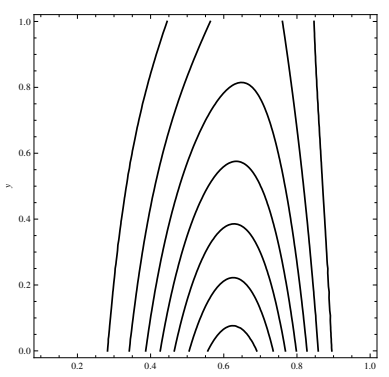

(d) D3D7 \& $\ell=2$

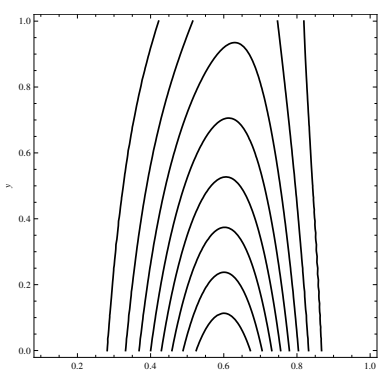

(g) D3D7 \& $\ell=3$

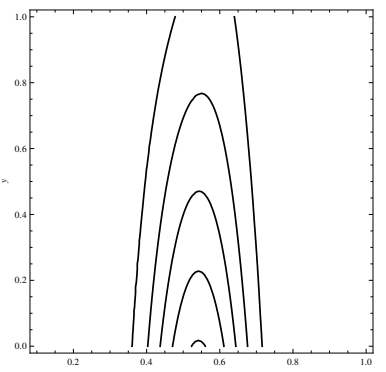

(j) $\mathrm{D} 3 \mathrm{D} 7 \& \ell=10$

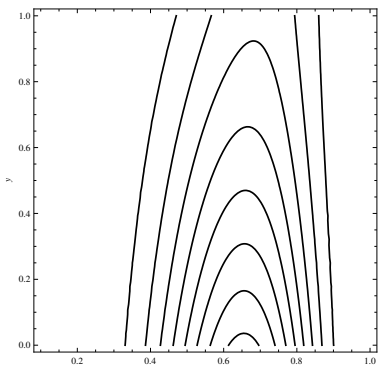

(b) $\mathrm{D} 4 \mathrm{D} 6 \overline{\mathrm{D} 6} \& \ell=1$

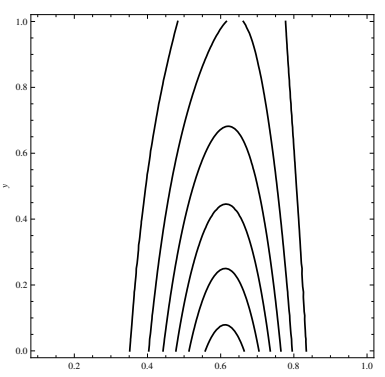

(e) $\mathrm{D} 4 \mathrm{D} 6 \overline{\mathrm{D} 6} \& \ell=2$

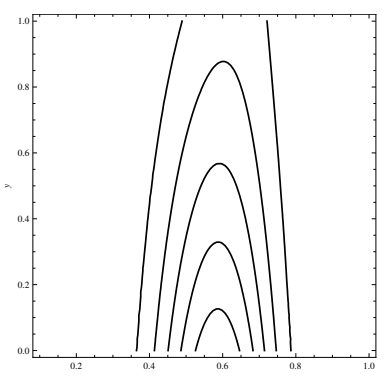

(h) $\mathrm{D} 4 \mathrm{D} 6 \overline{\mathrm{D}} 6 \& \ell=3$

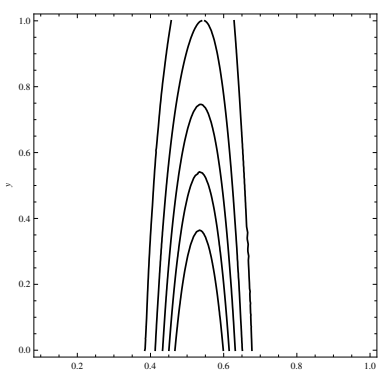

(k) $\mathrm{D} 4 \mathrm{D} 6 \overline{\mathrm{D} 6} \& \ell=10$

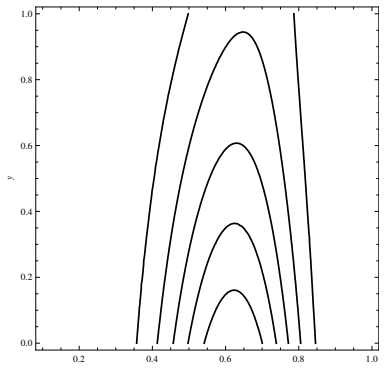

(c) $\mathrm{D} 4 \mathrm{D} 8 \overline{\mathrm{D} 8} \& \ell=1$

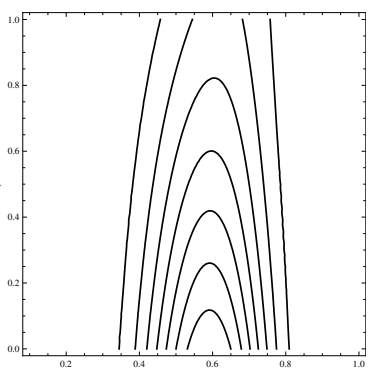

(f) $\mathrm{D} 4 \mathrm{D} 8 \overline{\mathrm{D} 8} \& \ell=2$

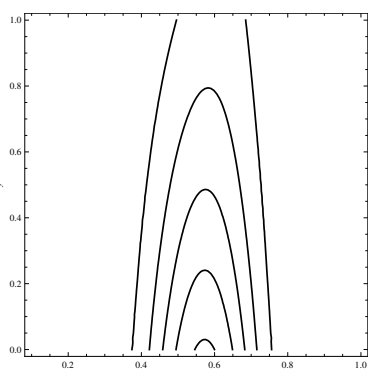

(i) $\mathrm{D} 4 \mathrm{D} 8 \overline{\mathrm{D} 8} \& \ell=3$

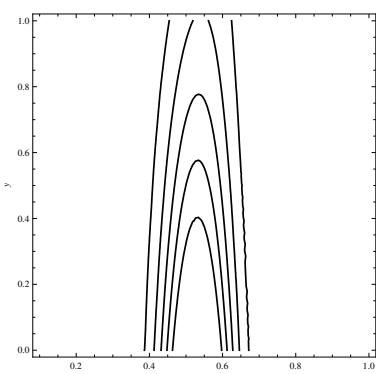

(l) $\mathrm{D} 4 \mathrm{D} 8 \overline{\mathrm{D} 8} \& \ell=10$

Figure 6. Contour line maps of DIS differential cross section for unpolarized holographic vector mesons for the three flavor-brane models we consider, in the region $1 / \sqrt{\lambda} \ll x<1$. Different mesons are labeled with $\ell=1,2,3$ and 10 (displayed on the four rows). The differential cross sections have been normalized as explained in the main text. The horizontal axis represents the Bjorken parameter within the range $[0.1,1]$, while the vertical one corresponds to the variable $y$, $[0,1]$. Broader curves correspond to lower values of the DIS cross section. 


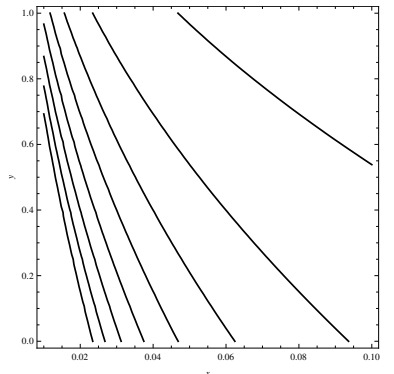

(a) D3D7 \& $\ell=1$

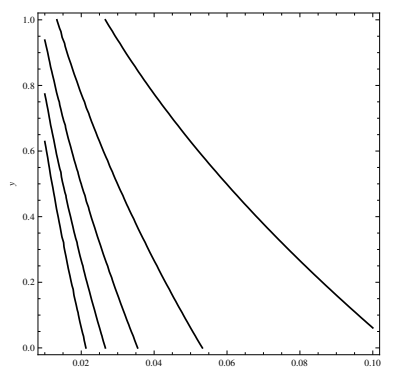

(d) D3D7 \& $\ell=2$

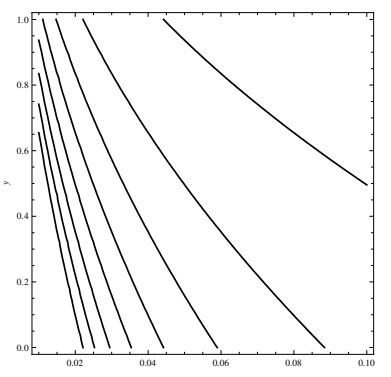

(g) D3D7 \& $\ell=3$

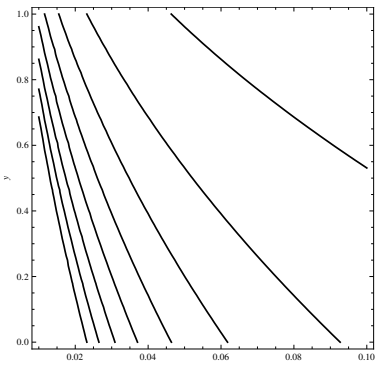

(j) D3D7 \& $\ell=10$

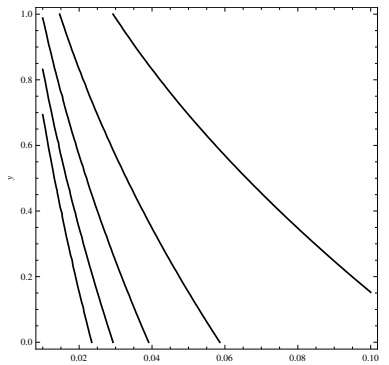

(b) $\mathrm{D} 4 \mathrm{D} 6 \overline{\mathrm{D} 6} \& \ell=1$

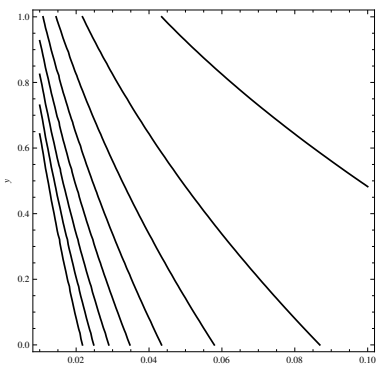

(e) $\mathrm{D} 4 \mathrm{D} 6 \overline{\mathrm{D} 6} \& \ell=2$

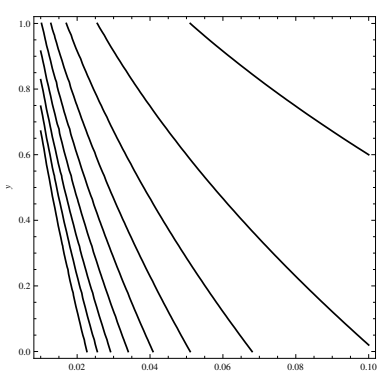

(h) $\mathrm{D} 4 \mathrm{D} 6 \overline{\mathrm{D} 6} \& \ell=3$

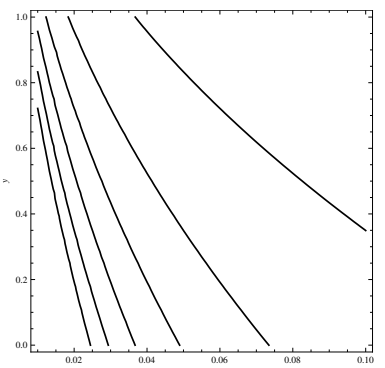

(k) $\mathrm{D} 4 \mathrm{D} 6 \overline{\mathrm{D} 6} \& \ell=10$

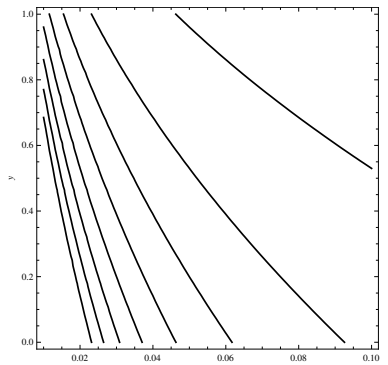

(c) $\mathrm{D} 4 \mathrm{D} 8 \overline{\mathrm{D} 8} \& \ell=1$

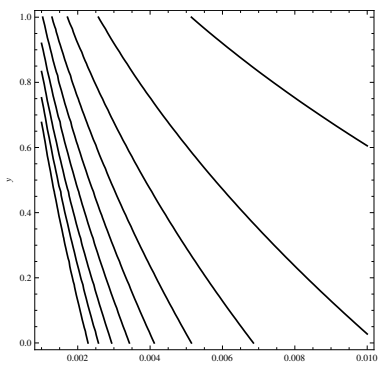

(f) $\mathrm{D} 4 \mathrm{D} 8 \overline{\mathrm{D} 8} \& \ell=2$

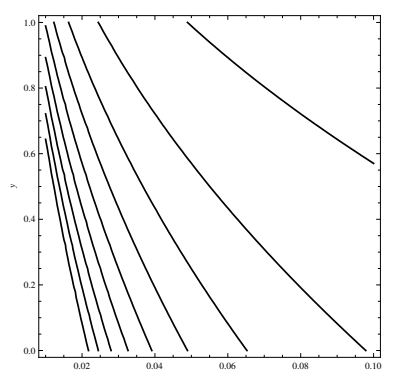

(i) $\mathrm{D} 4 \mathrm{D} 8 \overline{\mathrm{D} 8} \& \ell=3$

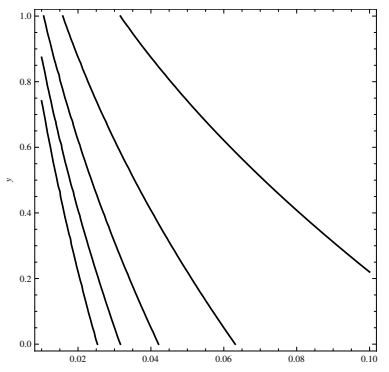

(l) $\mathrm{D} 4 \mathrm{D} 8 \overline{\mathrm{D} 8} \& \ell=10$

Figure 7. Contour line maps of DIS differential cross section for unpolarized holographic vector mesons for the three flavor-brane models we consider, in the region $\exp (-\sqrt{\lambda}) \ll x \ll 1 / \sqrt{\lambda}$. Different mesons are labeled with $\ell=1,2,3$ and 10 (displayed on the four rows). The differential cross sections have been normalized as explained in the main text. The horizontal axis represents the Bjorken parameter within the range [0.01, 0.1], while the vertical one corresponds to the variable $y,[0,1]$. Curves with larger slope correspond to larger values of the DIS cross section. 
Notice that in our calculations the pion corresponds to the $\ell=1$ meson in the D3D7model, since it is the lightest pseudoscalar particle. These phenomenological studies could in principle be compared with the results obtained in our previous papers studying the parametric range $1 / \sqrt{\lambda} \ll x<1[1,2]$. The small- $x$ behavior we find is difficult to compare with the existing phenomenological models. However, in [17] it was proposed a model in which the parton distribution functions have a sea term that diverges as $x \rightarrow 0$, as it occurs for our structure functions. In addition, this type of divergences beyond the valence term was shown to appear on the pion contribution to the deuteron structure function $b_{1}$ (see for instance figure 3 in reference [18]).

In general terms, our approach shares fundamental concepts with references $[9,19-$ 22 ] as well as it involves somehow similar techniques to the ones used by them. Very interestingly, in those references there is agreement with baryon DIS data from H1 and ZEUS experiments at HERA. In fact in these references the objects of study are the glueball structure functions. Since the structure functions of scalar mesons behave similarly as those of glueballs for all values of the Bjorken parameter, we regard it as an indication suggesting that our predictions should be correct. Keeping in mind that the results for glueballs can be compared with experimental information from HERA, we would expect that our results for mesons (which at least for scalar ones are closely related to the glueball results of [7]) could be confirmed.

\subsection{Comparison with lattice QCD}

Now, let us focus on the comparison with lattice QCD results. Particularly, we can compare the lower moments of DIS structure functions of the pion and the $\rho$ meson obtained from lattice QCD [23] with our own predictions. The $n$-th moment of a generic function $h\left(x, q^{2}\right)$ is given by

$$
M_{n}[h] \equiv \int_{0}^{1} d x x^{n-1} h\left(x, q^{2}\right) .
$$

We consider the spin-dependent structure functions of the pion and $\rho$ mesons. In particular, the lattice QCD calculations in [23] have considered Wilson fermions and they have been carried out for three values of the quark mass. Therefore, it allows one to carry out an extrapolation to the chiral limit. The way we can compare our results with the lattice QCD ones for the pion is by using our expressions of the $F_{2}$ structure function ${ }^{18}$ for small and large $x$ values, and integrate them with appropriate lower and upper cut-offs. The former is integrated from $x=0.0001$ to $x=0.1$, while the latter is integrated from $x=0.1$ to $x=1$. Notice that within each structure function there is an undetermined constant containing $\Lambda, R, q^{2}$ and some other numbers. In fact, there is a different constant for each parametric regime of the Bjorken variable. Thus, for each model there are two undetermined constants. They are effectively two free parameters for each flavor-brane model, which are determined by carrying out the best fitting to the lattice QCD data for the moments $M_{i}\left(F_{2}\right)$ with $i=1,2,3$. The rest of the fittings are performed in the same way.

\footnotetext{
${ }^{18}$ For the pion we use $F_{2}$ and not $F_{1}$ because one of the features of the large $N$ limit is that the virtual photon strikes the entire hadron, thus $F_{1}=0$ in the large- $x$ region.
} 


\begin{tabular}{|c|c|c|c|}
\hline Model / Moment & $M_{1}\left(F_{2}\right)$ & $M_{2}\left(F_{2}\right)$ & $M_{3}\left(F_{2}\right)$ \\
\hline Lattice QCD & 0.3047 & 0.1180 & 0.0583 \\
\hline D3D7 & 0.3067 & 0.0962 & 0.0658 \\
\hline Percentage error & -0.6 & 18.5 & -12.8 \\
\hline $\mathrm{D} 4 \mathrm{D} 8 \overline{\mathrm{D} 8}$ & 0.3061 & 0.1018 & 0.0643 \\
\hline Percentage error & -0.5 & 13.7 & -10.3 \\
\hline$\overline{\mathrm{D} 4 \mathrm{D} 6 \overline{\mathrm{D}} 6}$ & 0.3064 & 0.0990 & 0.0650 \\
\hline Percentage error & -0.6 & 16.1 & -11.6 \\
\hline
\end{tabular}

Table 3. Comparison of our results for the first moments of the structure function $F_{2}$ of the pion for a suitable choice of the normalization constants with respect to the average results of the lattice QCD computations in [23]. Uncertainties in the lattice computations are omitted.

Our results and the lattice QCD data as well as the deviations from each other are listed in table 3 . The constants of the small- $x$ regime which leads to the best fitting to the lattice QCD data of reference [23] are 0.025, 0.020 and 0.022, while for the large- $x$ regime we found 24, 2020 and 267, for the D3D7-, the D4D8 $\overline{\mathrm{D} 8}$ - and the D4D6 $\overline{\mathrm{D} 6}$-brane models, respectively. We find discrepancies with respect to the lattice QCD calculations up to $18 \%$ for our results in the case of the pion, $\ell=1$. Recall that in the $1 / N$ expansion discrepancies are of order $30 \%$, thus our results are within the expectations. We should keep in mind that when analyzing discrepancies between gauge/string duality predictions, phenomenology and experimental data, for instance bottom-up models in five dimensions (the AdS/QCD model) give results related to masses and decay constants of mesons with discrepancies of order $5 \%[24,25]$. On the other hand, for the case of more involved calculations leading to the $\Delta I=1 / 2$ rule describing the kaon decays, related to the calculation of four-point correlation functions, discrepancies become of order $30 \%$ [26, 27].

There are more recent results in the lattice QCD literature to compare with. For instance we can consider data from references [28, 29]. The results for these fittings are shown in table 4 . One can see that all the results are improved being the deviation smaller than $10 \%$. In this case, the constants we find are $0.014,0.011$ and 0.012 for the small- $x$ regime, and 29, 2450 and 325 for the large- $x$ regime, for the D3D7-, the $\mathrm{D} 4 \mathrm{D} 8 \overline{\mathrm{D}} 8$ - and the D4D6 $\overline{\mathrm{D} 6}$-brane models, respectively.

It is possible to understand a bit better the comparison between lattice QCD data and our holographic results by isolating the contributions from the small- $x$ and large- $x$ structure functions to each moment. We are tempted to associate the small- $x$ region with the concept of sea-quark distribution function, which describes the possibility of finding a quark or an anti-quark (generated by gluon splitting) carrying a very small fraction of the hadron momentum. In this picture the large- $x$ structure function is then related to the valence distribution function associated with quarks that carry a considerable fraction of the hadron momentum. ${ }^{19}$ Table I in [30] shows that both for lattice and for phenomenological results one finds that the contribution of the sea of quarks is important only for the first moment of the structure functions, since it yields a considerable fraction of the final result. In the

\footnotetext{
${ }^{19}$ These are not formal definitions of these concepts.
} 


\begin{tabular}{|c|c|c|c|}
\hline Model / Moment & $M_{1}\left(F_{2}\right)$ & $M_{2}\left(F_{2}\right)$ & $M_{3}\left(F_{2}\right)$ \\
\hline Lattice QCD & 0.27 & 0.13 & 0.074 \\
\hline D3D7 & 0.2708 & 0.1161 & 0.0803 \\
\hline Percentage error & -0.3 & 10.7 & -8.5 \\
\hline $\mathrm{D} 4 \mathrm{D} 8 \overline{\mathrm{D} 8}$ & 0.2705 & 0.1221 & 0.0779 \\
\hline Percentage error & -0.2 & 6.1 & -5.2 \\
\hline $\mathrm{D} 4 \mathrm{D} 6 \overline{\mathrm{D} 6}$ & 0.2706 & 0.1191 & 0.0791 \\
\hline Percentage error & -0.2 & 8.4 & -6.9 \\
\hline
\end{tabular}

Table 4. Comparison of our results for the first moments of the structure function $F_{2}$ for the pion for a suitable choice of the normalization constants with respect to the average results of the lattice QCD computations in $[28,29]$. Uncertainties in the lattice computations are omitted.

\begin{tabular}{|c|c|c|c|}
\hline Model / Moment & $M_{2}\left(F_{1}\right)$ & $M_{3}\left(F_{1}\right)$ & $M_{4}\left(F_{1}\right)$ \\
\hline Lattice QCD & 0.1743 & 0.074 & 0.035 \\
\hline D3D7 & 0.1755 & 0.059 & 0.040 \\
\hline Percentage error & -0.7 & 21.3 & -14.1 \\
\hline $\mathrm{D} 4 \mathrm{D} 8 \overline{\mathrm{D} 8}$ & 0.1752 & 0.062 & 0.040 \\
\hline Percentage error & -0.5 & 16.4 & -11.8 \\
\hline$\overline{\mathrm{D} 4 \mathrm{D} 6 \overline{\mathrm{D}} 6}$ & 0.1754 & 0.060 & 0.040 \\
\hline Percentage error & -0.6 & 18.8 & -13.0 \\
\hline
\end{tabular}

Table 5. Comparison of our results for the first moments of the structure function $F_{1}$ for the $\rho$ meson for a suitable choice of the normalization constants with respect to the average results of the lattice QCD computations in [23]. Uncertainties in the lattice computations are omitted.

second and third moments its contribution is substantially reduced, and the contribution from the valence distribution function gives almost the full result. This is similar to what happens with our structure functions: the integral for the small- $x$ region is important in order to fit the first moment $M_{1}$, but one can almost ignore it for $M_{2}$ and $M_{3}$.

Table 5 shows the comparison of our results for the first moments of the structure function $F_{1}$ for the $\rho$ meson for a suitable choice of the normalization constants with respect to the average results of the lattice QCD computations in [23]. The constants in this case are $0.013,0.011$ and 0.012 for the small- $x$ regime, and 14, 1229 and 162 for the large- $x$ regime, for the D3D7-, the $\mathrm{D} 4 \mathrm{D} 8 \overline{\mathrm{D} 8}$ - and the $\mathrm{D} 4 \mathrm{D} 6 \overline{\mathrm{D} 6}$-brane models, respectively. As it was commented for table 3 , it is very interesting that the relative discrepancies are smaller than 21 percent. Now, by using tables 4 and 5 we can draw figures 8 and 9 . In figure 8 the first three moments for $F_{2}$ are displayed for the pion. The free parameters for each dual holographic model are chosen in order to fit the results in [23] obtained with lattice QCD as explained before. The three studied holographic dual models are shown, together with the value obtained in the reference. We find good agreement with lattice QCD data with discrepancies lower than 20\%. By using more recent lattice QCD results of references $[28,29]$ the fittings improve with discrepancies lower than $10 \%$. In addition, in figure 9 the first three moments of $F_{1}$ are shown for the $\rho$ meson. The three holographic dual models are presented in a similar way as in figure 8 . Discrepancies with respect to 


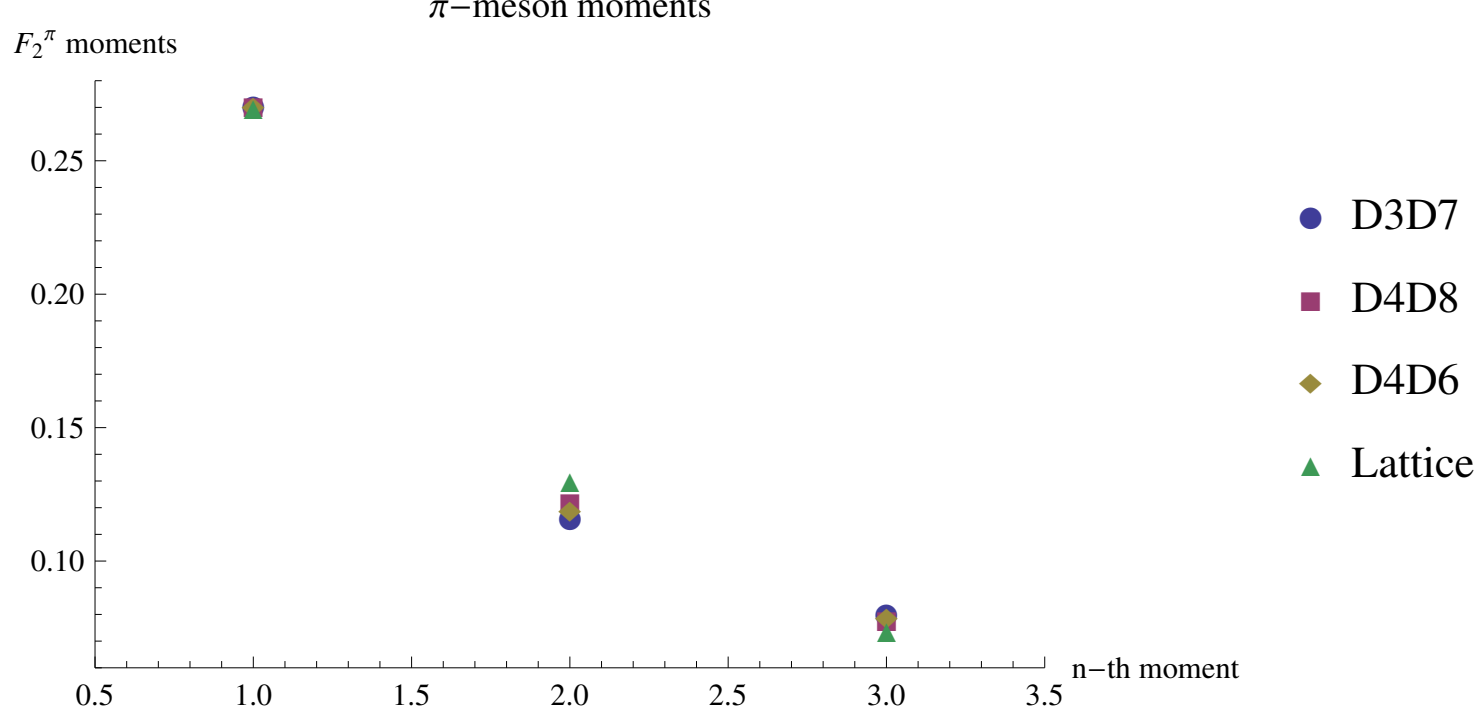

Figure 8. The first three moments of $F_{2}$ are shown for the $\pi$ meson. The free parameters for each dual holographic model are chosen in order to fit the results in [23], obtained with lattice QCD as explained before. The three holographic dual models are shown, along with the average value obtained in the mentioned reference. Discrepancies with respect to the reference are under $20 \%$.

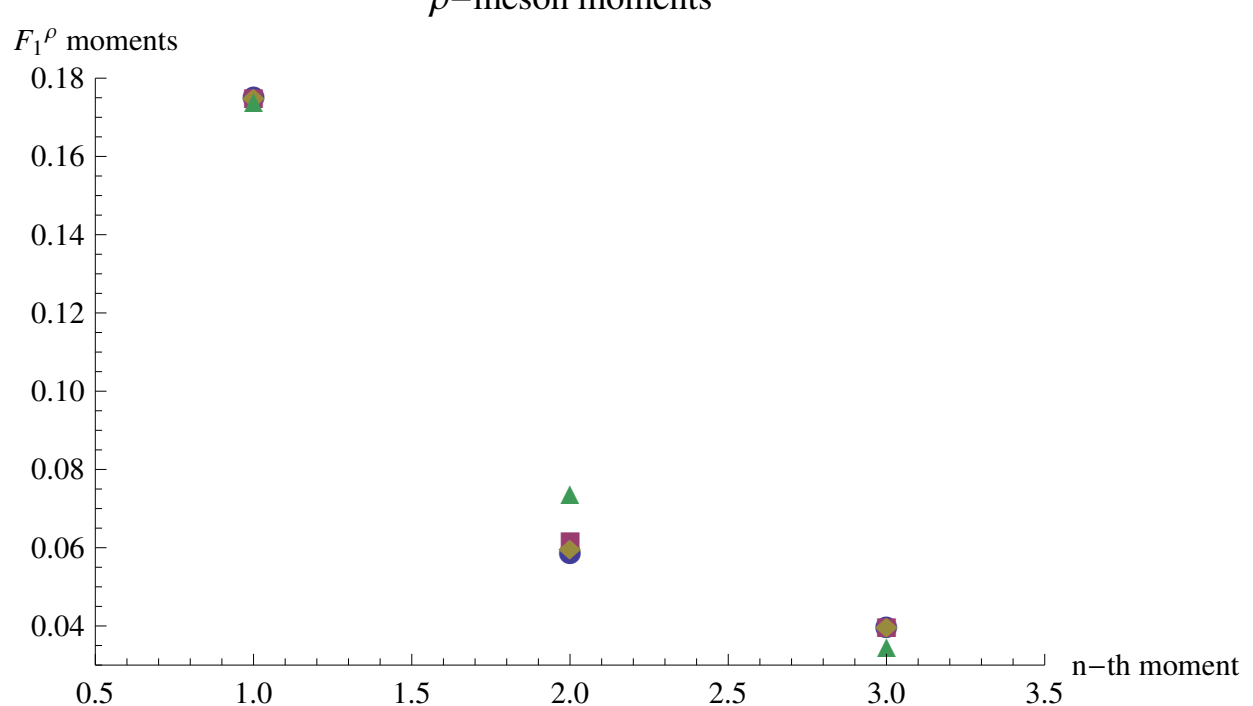

- D3D7

- D4D8

- D4D6

$\triangle$ Lattice

Figure 9. The first three moments of $F_{1}$ are shown for the $\rho$ meson. The free parameters for each holographic dual model are chosen in order to fit the results in [23], obtained with lattice QCD. The three holographic dual models are shown, along with the average value obtained in this reference. Discrepancies with respect to the reference are under $21 \%$ in all cases. 
that reference are under $21 \%$ in all cases. Therefore, this also shows good agreement with lattice QCD data.

Notice that in all our fittings the constant of the structure function corresponding to the small- $x$ regime is always a few orders of magnitude smaller than the one associated with the large- $x$ structure function. This is in full agreement with our calculations, at least in the regime where the holographic dual model represents an accurate description of the dual gauge theory. This could have been directly predicted from our analytical results. By examining the difference between the constants in front of $(4.5)$ and $(4.18,4.19)$ one can see that for the $\ell=1$ scalar meson the numerical coefficients and normalization constants are of the same order, and the main difference is given by the $\lambda^{-1 / 2}$ factor that appears in the small- $x$ case. This is small at large 't Hooft coupling, and it is directly related to the value of $x=0.1$ which we use in order to separate the first two regimes (A and $\mathrm{B}$ ) in our calculations.

Another important point comes also from the comparison between our analytical results and the different models formulated in the literature in order to fit the phenomenological data for the measurement of the pion valence distribution functions. As it is explained in $[30,31]$ there have been different attempts to describe the available data, and while there are some differences between them, they all agree on the fact that the distribution functions should behave as $(1-x)^{2}$ in the $x \rightarrow 1$ limit. In [32] it has been found a fall-off $\left(\sim(1-x)^{2 \pm 0.1}\right)$ for the valence distribution function consistent with Drell-Yan data. This is consistent with theoretical predictions based on perturbative QCD (see for instance [33] and references therein) as well as with calculations using Dyson-Schwinger equations [34]. This is exactly what happens with our structure functions (4.5) in the D3D7-brane model for the $\ell=1$ scalar meson, i.e. the pion. This would suggest that the D3D7-brane model gives better predictions than the other two ones studied in this work. In the $\mathrm{D} 4 \mathrm{D} 8 \overline{\mathrm{D}} 8$-brane and $\mathrm{D} 4 \mathrm{D} 6 \overline{\mathrm{D} 6}$-brane models the exponents are 4.59 and 3.33 , respectively.

A very interesting review of lattice QCD calculations on moments of hadron distribution functions, as well as other important hadron structure observables is given in [35]. The earliest calculations of the lowest two moments of the pion quark distribution function have been done in [36-38] in quenched lattice QCD. An extensive study of several moments of the pion and the $\rho$ meson have been done in [23]. In fact, we have compared our results with those in that reference in our tables 3 and 5 and in our figures 8 and 9 , while in our table 4 we compare with references [28, 29]. The earliest results of [23] have been obtained by using quenched lattice QCD simulations, which means that the fermion determinant is set to one, thus neglecting all quark-antiquark quantum fluctuations. This introduces an error which can only be quantified by comparison with dynamical simulations with physical quark masses, which are only computationally possible since a few years. ${ }^{20}$ In fact for instance in $[28,29,31]$ dynamical quarks have been considered.

We may also try to use this kind of fittings to compare the first few moments corresponding to our $b_{i}$ functions with the ones calculated in [23]. However, in that paper the results found for these moments are smaller than the $F_{i}$ moments. ${ }^{21}$ Since the structure

\footnotetext{
${ }^{20}$ We thank Andreas Schäfer for this comment.

${ }^{21}$ In fact, even if we actually tried to do this the very small values of these moments and the considerable errors associated with them in [23] would probably make the fittings unreliable.
} 
functions we obtained from string theory satisfy the relations $b_{i}=3 F_{i}$, the numerical comparison becomes meaningless. It seems that there is a conceptual difference, and it would be very interesting to study where it comes from or to be able to resolve this discrepancy from new experimental data.

Once again we would like to emphasize that the discussion presented in this last section has to be understood qualitatively. One should keep in mind that the gauge theories that we are able to study in terms of string theory dual models do not look exactly like QCD. What makes them interesting though is that they have some important similarities related to the meson spectrum and the concepts of color confinement, chiral symmetry breaking and other properties. Thus, it is really interesting that these holographic dual models give predictions of the moments of the structure functions which are comparable to lattice QCD results.

The good agreement we have obtained with lattice QCD data is somehow not unexpected taking into account several examples where the supergravity dual models give results which compare reasonably well with lattice QCD data. For instance, in the case of SYM plasmas good level of agreement has been found between lattice QCD results and the gauge/gravity duality approach by including string theory corrections to the supergravity calculations as it has been shown for the mass and electric charge transport coefficients. In the case of the mass transport the quantitative agreement between lattice QFT calculations for $\eta / s[39]$ and the gauge/string duality [40,41] is remarkable $(\eta$ is the $\mathcal{N}=4 \mathrm{SYM}$ plasma shear viscosity and $s$ the entropy density). Also for electric charge transport the DC conductivity has been calculated using lattice QCD [42] and the gauge/string duality [43], including $\mathcal{O}\left(\alpha^{\prime 3}\right)$ corrections, leading to good level of agreement [44-47]. Of course, also in those cases the gauge/string dual calculations strictly hold for large $N$ and the strong coupling expansion $(1 / \lambda)$, but if one insists on using $N=3$ and $\lambda \approx 15$ it leads to the results we mention in this paragraph.

Also, the fact that the methods developed for the holographic dual description of DIS can be extended to other strongly coupled $\mathrm{SU}(N)$ gauge theories is very interesting.

\section{Acknowledgments}

We would like to thank Sergio Iguri, José Goity, Carlos Núñez and Andreas Schäfer for very valuable comments on the manuscript. The work of E.K., N.K. and M.S. is supported by the CONICET. This work has been partially supported by the CONICET-PIP 0595/13 the grant and UNLP grant 11/X648.

Open Access. This article is distributed under the terms of the Creative Commons Attribution License (CC-BY 4.0), which permits any use, distribution and reproduction in any medium, provided the original author(s) and source are credited.

\section{References}

[1] E. Koile, S. Macaluso and M. Schvellinger, Deep Inelastic Scattering from Holographic Spin-One Hadrons, JHEP 02 (2012) 103 [arXiv:1112.1459] [INSPIRE]. 
[2] E. Koile, S. Macaluso and M. Schvellinger, Deep inelastic scattering structure functions of holographic spin-1 hadrons with $N_{f} \geq 1$, JHEP 01 (2014) 166 [arXiv:1311.2601] [INSPIRE].

[3] E. Koile, N. Kovensky and M. Schvellinger, Hadron structure functions at small $x$ from string theory, JHEP 05 (2015) 001 [arXiv:1412.6509] [INSPIRE].

[4] M. Kruczenski, D. Mateos, R.C. Myers and D.J. Winters, Meson spectroscopy in AdS/CFT with flavor, JHEP 07 (2003) 049 [hep-th/0304032] [INSPIRE].

[5] M. Kruczenski, D. Mateos, R.C. Myers and D.J. Winters, Towards a holographic dual of large $N_{c} Q C D$, JHEP 05 (2004) 041 [hep-th/0311270] [INSPIRE].

[6] T. Sakai and S. Sugimoto, Low energy hadron physics in holographic QCD, Prog. Theor. Phys. 113 (2005) 843 [hep-th/0412141] [INSPIRE].

[7] J. Polchinski and M.J. Strassler, Deep inelastic scattering and gauge/string duality, JHEP 05 (2003) 012 [hep-th/0209211] [INSPIRE].

[8] J. Polchinski and M.J. Strassler, The string dual of a confining four-dimensional gauge theory, hep-th/0003136 [INSPIRE].

[9] R.C. Brower, J. Polchinski, M.J. Strassler and C.-I. Tan, The Pomeron and gauge/string duality, JHEP 12 (2007) 005 [hep-th/0603115] [INSPIRE].

[10] A.V. Manohar, An Introduction to spin dependent deep inelastic scattering, hep-ph/9204208 [INSPIRE].

[11] P. Hoodbhoy, R.L. Jaffe and A. Manohar, Novel Effects in Deep Inelastic Scattering from Spin 1 Hadrons, Nucl. Phys. B 312 (1989) 571 [INSPIRE].

[12] C.A. Ballon Bayona, H. Boschi-Filho, N.R.F. Braga and M.A.C. Torres, Deep inelastic scattering for vector mesons in holographic D4-D8 model, JHEP 10 (2010) 055 [arXiv: 1007.2448] [INSPIRE].

[13] K. Wijesooriya, P.E. Reimer and R.J. Holt, The pion parton distribution function in the valence region, Phys. Rev. C 72 (2005) 065203 [nucl-ex/0509012] [INSPIRE].

[14] R.J. Holt and C.D. Roberts, Distribution Functions of the Nucleon and Pion in the Valence Region, Rev. Mod. Phys. 82 (2010) 2991 [arXiv:1002.4666] [InSPIRE].

[15] P.E. Reimer, R.J. Holt and K. Wijesooriya, The partonic structure of the pion at large-x, AIP Conf. Proc. 1369 (2011) 153 [InSPIRE].

[16] L. Chang and A.W. Thomas, Pion Valence-quark Parton Distribution Function, Phys. Lett. B 749 (2015) 547 [arXiv:1410.8250] [InSPIRE].

[17] G. Altarelli, S. Petrarca and F. Rapuano, The pion structure function in a constituent model, Phys. Lett. B 373 (1996) 200 [hep-ph/9510346] [INSPIRE].

[18] G.A. Miller, Pionic and Hidden-Color, Six-Quark Contributions to the Deuteron b1 Structure Function, Phys. Rev. C 89 (2014) 045203 [arXiv:1311.4561] [InSPIRE].

[19] R.C. Brower, M.J. Strassler and C.-I. Tan, On the eikonal approximation in AdS space, JHEP 03 (2009) 050 [arXiv: 0707.2408] [INSPIRE].

[20] R.C. Brower, M. Djuric, I. Sarcevic and C.-I. Tan, String-Gauge Dual Description of Deep Inelastic Scattering at Small-x, JHEP 11 (2010) 051 [arXiv:1007.2259] [INSPIRE].

[21] R.C. Brower, M. Djuric, I. Sarcevic and C.-I. Tan, Small-x Deep Inelastic Scattering via the Pomeron in AdS, arXiv:1204.0472 [INSPIRE]. 
[22] R. Brower, R.C. Brower, M. Djurić, T. Raben and C.-I. Tan, Towards holographic QCD: AdS/CFT, confinement deformation and DIS at small-x, arXiv:1412.3443 [INSPIRE].

[23] C. Best et al., Pion and rho structure functions from lattice QCD, Phys. Rev. D 56 (1997) 2743 [hep-lat/9703014] [INSPIRE].

[24] J. Erlich, E. Katz, D.T. Son and M.A. Stephanov, QCD and a holographic model of hadrons, Phys. Rev. Lett. 95 (2005) 261602 [hep-ph/0501128] [INSPIRE].

[25] L. Da Rold and A. Pomarol, Chiral symmetry breaking from five dimensional spaces, Nucl. Phys. B 721 (2005) 79 [hep-ph/0501218] [INSPIRE].

[26] T. Hambye, B. Hassanain, J. March-Russell and M. Schvellinger, Four-point functions and Kaon decays in a minimal AdS/QCD model, Phys. Rev. D 76 (2007) 125017 [hep-ph/0612010] [INSPIRE].

[27] T. Hambye, B. Hassanain, J. March-Russell and M. Schvellinger, On the Delta $I=1 / 2$ rule in holographic QCD, Phys. Rev. D 74 (2006) 026003 [hep-ph/0512089] [InSPIRE].

[28] D. Brommel et al., Quark distributions in the pion, PoS(LATTICE 2007)140.

[29] L. Chang, C. Mezrag, H. Moutarde, C.D. Roberts, J. Rodríguez-Quintero and P.C. Tandy, Basic features of the pion valence-quark distribution function, Phys. Lett. B 737 (2014) 23 [arXiv:1406.5450] [INSPIRE].

[30] W. Detmold, W. Melnitchouk and A.W. Thomas, Parton distribution functions in the pion from lattice QCD, Phys. Rev. D 68 (2003) 034025 [hep-lat/0303015] [InSPIRE].

[31] M. Aicher, A. Schafer and W. Vogelsang, Threshold-Resummed Cross section for the Drell-Yan Process in Pion-Nucleon Collisions at COMPASS, Phys. Rev. D 83 (2011) 114023 [arXiv: 1104.3512] [INSPIRE].

[32] M. Aicher, A. Schafer and W. Vogelsang, Soft-gluon resummation and the valence parton distribution function of the pion, Phys. Rev. Lett. 105 (2010) 252003 [arXiv:1009.2481] [INSPIRE].

[33] F. Yuan, Generalized parton distributions at $x \rightarrow 1$, Phys. Rev. D 69 (2004) 051501 [hep-ph/0311288] [INSPIRE].

[34] M.B. Hecht, C.D. Roberts and S.M. Schmidt, Valence quark distributions in the pion, Phys. Rev. C 63 (2001) 025213 [nucl-th/0008049] [INSPIRE].

[35] P. Hagler, Hadron structure from lattice quantum chromodynamics, Phys. Rept. 490 (2010) 49 [arXiv: 0912.5483] [inSPIRE].

[36] G. Martinelli and C.T. Sachrajda, Pion Structure Functions From Lattice QCD, Phys. Lett. B 196 (1987) 184 [INSPIRE].

[37] G. Martinelli and C.T. Sachrajda, A Lattice Calculation of the Second Moment of the Pion's Distribution Amplitude, Phys. Lett. B 190 (1987) 151 [InSPIRE].

[38] G. Martinelli and C.T. Sachrajda, A Lattice Calculation of the Pion's Form-Factor and Structure Function, Nucl. Phys. B 306 (1988) 865 [inSPIRE].

[39] H.B. Meyer, A calculation of the shear viscosity in $\mathrm{SU}(3)$ gluodynamics, Phys. Rev. D 76 (2007) 101701 [arXiv:0704.1801] [INSPIRE].

[40] P. Kovtun, D.T. Son and A.O. Starinets, Viscosity in strongly interacting quantum field theories from black hole physics, Phys. Rev. Lett. 94 (2005) 111601 [hep-th/0405231] [INSPIRE]. 
[41] R.C. Myers, M.F. Paulos and A. Sinha, Quantum corrections to $\eta / s$, Phys. Rev. D 79 (2009) 041901 [arXiv:0806.2156] [INSPIRE].

[42] G. Aarts, C. Allton, J. Foley, S. Hands and S. Kim, Spectral functions at small energies and the electrical conductivity in hot, quenched lattice QCD, Phys. Rev. Lett. 99 (2007) 022002 [hep-lat/0703008] [INSPIRE].

[43] S. Caron-Huot, P. Kovtun, G.D. Moore, A. Starinets and L.G. Yaffe, Photon and dilepton production in supersymmetric Yang-Mills plasma, JHEP 12 (2006) 015 [hep-th/0607237] [INSPIRE].

[44] B. Hassanain and M. Schvellinger, Diagnostics of plasma photoemission at strong coupling, Phys. Rev. D 85 (2012) 086007 [arXiv:1110.0526] [INSPIRE].

[45] B. Hassanain and M. Schvellinger, Plasma conductivity at finite coupling, JHEP 01 (2012) 114 [arXiv:1108.6306] [INSPIRE].

[46] B. Hassanain and M. Schvellinger, Towards 't Hooft parameter corrections to charge transport in strongly-coupled plasma, JHEP 10 (2010) 068 [arXiv:1006.5480] [INSPIRE].

[47] B. Hassanain and M. Schvellinger, Plasma photoemission from string theory, JHEP 12 (2012) 095 [arXiv:1209.0427] [InSPIRE]. 